\title{
(E)-2-(4-Arylbut-1-en-3-yn-1-yl)chromones as Synthons for the Synthesis of Xanthone-1,2,3-triazole Dyads
}

\author{
Hélio M. T. Albuquerque, ${ }^{[a]}$ Clementina M. M. Santos, ${ }^{[a, b]}$ José A. S. Cavaleiro, ${ }^{[a]}$ and \\ Artur M. S. Silva*[a]
}

\begin{abstract}
Keywords: Cycloaddition / Diels-Alder reaction / Cyclization / Click chemistry / Oxygen heterocycles / Nitrogen heterocycles
\end{abstract}

Xanthone-1,2,3-triazole dyads have been synthesized by two different approaches, both starting from novel (E)-2-(4-arylbut-1-en-3-yn-1-yl)chromones, prepared through a base-catalyzed aldol reaction of 2-methylchromone and arylpropargyl aldehydes. In the first method, the xanthone moiety is built by Diels-Alder reaction of the referred unsaturated chromones with $\mathrm{N}$-methylmaleimide under microwave irradiation, followed by oxidation of the obtained adducts with
DDQ, whereas the 1,2,3-triazole ring results from the cycloaddition reaction of the acetylene moiety with sodium azide. The second strategy first involves the cycloaddition reaction with sodium azide to provide the 1,2,3-triazole ring, followed by methylation of the triazole $\mathrm{NH}$ group prior to Diels-Alder reaction with $N$-methylmaleimide. The last step in this synthesis of novel xanthone-1,2,3-triazole dyads entails oxidation of the cycloadducts with DDQ.

\section{Introduction}

Xanthones or $9 H$-xanthen-9-ones are one of the most important classes of naturally occurring oxygenated heterocyclic compounds possessing a dibenzo- $\gamma$-pyrone framework. The parent xanthone has not been reported as a natural product. ${ }^{[1]}$ However, in 2002 Oldenburg et al. described the occurrence of this xanthone in crude oils from offshore Norway. The authors suggest that this product can be formed by oxidation of xanthene in the reservoir or obtained by geosynthesis from aromatic precursors. ${ }^{[2]}$ Natural xanthones often appear as highly substituted derivatives, bearing methoxy, hydroxy, alkyl, isopentenyl and glycosyl groups in their monomeric, dimeric, polycyclic or xanthonolignoide forms. ${ }^{[3]}$ In the past few years, a great number of studies have emphasized the biological and pharmacological properties of both natural and synthetic xanthones, ${ }^{[4]}$ including anti-inflammatory, ${ }^{[5]}$ cancer chemopreventive, ${ }^{[6]}$ antimalarial, ${ }^{[7]}$ and radical scavenging activities. ${ }^{[8]}$ The inhibitory activities of these agents against enzymes such as cyclooxygenase and cholinesterase have also been reported. ${ }^{[4,9]}$

Likewise, among the nitrogen heterocycles, triazoles have particular relevance due to their application in several research fields such as biochemistry, pharmaceutical and ma-

[a] Department of Chemistry \& QOPNA, University of Aveiro, Campus de Santiago, 3810-193 Aveiro, Portugal

E-mail: artur.silva@ua.pt

https://sites.google.com/site/artursilvaua/silva-ams

[b] School of Agriculture, Polytechnic Institute of Bragança, Campus de Santa Apolónia, 5301-855 Bragança, Portugal

Supporting information for this article is available on the WWW under http://dx.doi.org/10.1002/ejoc.201500448. terial science. Triazoles have been used as drugs, ${ }^{[10]}$ and are known to possess anti-HIV-type I protease, ${ }^{[11]}$ anti-hyperglycemic, ${ }^{[12]}$ and antimicrobial activities, among others. ${ }^{[13]}$ In terms of medicinal chemistry, it has been demonstrated that 1,4- and 1,5-disubstituted 1,2,3-triazoles can participate in important binding interactions with biological targets, maintaining a good pharmacokinetic profile. ${ }^{[14]}$ In addition, they are commercially used as anticorrosive agents, ${ }^{[15]}$ agrochemicals, ${ }^{[16]}$ photostabilizers and dyes. ${ }^{[17]}$

Considering the biological properties exhibited by these two classes of heterocycles, the development of new production methods for xanthone-1,2,3-triazole dyads aimed specifically at developing potentially improved therapeutic agents is a high priority. This idea was recently exemplified by a study carried out by Zou et al. in which xanthones bearing a 1,4-disubstituted-1,2,3-triazole moiety showed promising antitumor activity. ${ }^{[18]}$ Another study indicated that 3,6-dihydroxyxanthone, known as a good fluorophore, increased its fluorescence upon triazole formation. ${ }^{[19]}$ With this rational in mind, we designed and synthesized novel chromone derivatives to be used as building blocks in the synthesis of xanthone-1,2,3-triazole dyads. The first reports dealing with the reactivity of chromone derivatives in DielsAlder (DA) reactions en route to xanthone derivatives have been summarized by our group in 1993. ${ }^{[20]}$ At that time, xanthene adducts were not characterized and were assumed to have the expected 1,2,3,9a-tetrahydroxanthene structure. Subsequently, other studies revealed that the products formed were, in fact, 1,2,3,4-tetrahydroxanthenes. ${ }^{[21]}$ In 2000, 2-vinylchromones were also used as starting materials in [4 +2] cycloaddition reactions with enamines for the synthesis of new xanthone derivatives. ${ }^{[22]}$ More recently, studies 
on DA reactions of 3-styrylchromones under microwave irradiation in solvent-free conditions resulted in a new strategy for the synthesis of novel xanthones. ${ }^{[23]}$ In 2009, 2- and 3 -styrylchromones were used in cyclization reactions to afford new polyhydroxylated 2,3-diarylxanthones. ${ }^{[24]}$ Following the previously described studies, 2-(4-arylbuta-1,3-dien1-yl)chromones underwent electrocyclization and oxidation processes in order to prepare 1-arylxanthones. ${ }^{[25]}$ In this paper, we disclose our synthesis of new chromone derivatives, 2-(4-arylbut-1-en-3-yn-1-yl)chromones $\mathbf{4 a - d}$, which were further used as synthons to generate new xanthone-1,2,3triazole dyads. Such chromones $4 \mathbf{a}-\mathbf{d}$ possess two unsaturated systems (a diene and an alkyne) anticipated to display different reactivities in cycloaddition reactions. Moreover, these new chromones can participate in two consecutive types of cycloaddition reactions; namely, DA and Huisgen azide-alkyne 1,3-dipolar cycloadditions ("click chemistry"). Herein, we present two different synthetic strategies enabling facile access to new chromone-1,2,3-triazole derivatives as well as xanthone-1,2,3-triazole dyads.

\section{Results and Discussion}

\section{Syntheses}

Novel (E)-2-(4-arylbut-1-en-3-yn-1-yl)chromones $4 \mathbf{4 a - d}$ were obtained in moderate to good yields (52-80\%) using a base-catalyzed aldol reaction of 2-methylchromone 1 with arylpropargyl aldehydes $\mathbf{3 a - d}$ in ethanolic solutions (Scheme 1). ${ }^{25]}$ The required starting material 2-methylchromone (1) was prepared by a three-step Baker-Venkataraman sequence in good overall yields. ${ }^{[26]}$ Since arylpropargyl aldehydes $\mathbf{3 b}$-d are not commercially available, we generated them using a two-step approach: i) palladiumcatalyzed cross-coupling reaction of aryl iodides with propargyl alcohol ${ }^{[27]}$ in order to prepare the corresponding arylpropargyl alcohols $\mathbf{2 b - d}$, in good yields (79-95\%); ii) oxid-

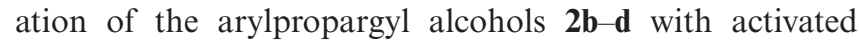
$\mathrm{MnO}_{2}$ to afford desired arylpropargyl aldehydes $\mathbf{3 b}-\mathbf{d}$, in excellent yields (86-97\%). The first step of this methodol- ogy was slightly improved by using toluene as solvent in the presence of 1.5 equiv. piperidine instead of in neat diethylamine (Scheme 1).

In our first approach to the target xanthone-1,2,3-triazole dyads, newly synthesized chromones $4 \mathbf{a}-\mathbf{d}$ were used as dienes in DA reactions with the poor dienophile $\mathrm{N}$ methyl-maleimide (Scheme 2). The structure of each conjugated diene suggests that it could not be very reactive due to extended electron delocalization. Thus, we tried highly energetic reaction conditions using 1,2,4-trichlorobenzene (TCB) under refluxing conditions (Table 1, Entry 1). No DA reaction took place and after a reaction time of $7 \mathrm{~d}$ only degradation of the starting material was observed when using two different derivatives $\mathbf{4 a}$ and $\mathbf{4 c}$ (Table 1, Entries 1 and 2). Slightly less energetic conditions (DMF at reflux for $48 \mathrm{~h}$ ) once again failed to produce any DA reaction despite the inclusion of the Lewis acid $\mathrm{Sc}(\mathrm{OTf})_{3}$ (Table 1, Entries 3 and 4). The failure of the normal (or "typical") electron demand DA reaction to produce meaningful results inspired us to attempt the reaction using an electron rich dienophile (dihydropyran) in neat conditions (Table 1, Entry 5). This DA reaction also failed to take place; we postulate that this is a consequence of the low reaction temperature imposed by the low boiling point of dihydropyran.

On the basis of previous findings by our group ${ }^{[23,28]}$ we performed the DA reaction under microwave irradiation (multimode apparatus) conditions. Several experiments were carried out in which reaction times, solvent, Lewis acids, amounts of dienophile and microwave potencies were varied (Table 1, Entries 6-15). The best results were achieved in a reaction time of $20 \mathrm{~min}$, at $160^{\circ} \mathrm{C}$ using a few drops of DMF as solvent (Table 1, Entries 6, 16-18). DA adducts 5a-d were obtained in low yields (20-30\%) although it is worth noting that a significant amount of starting chromone could be readily recovered $(65-70 \%)$. Thus, the poor yields can be explained by the competition of the retro-DA reaction, which is often promoted at such temperatures. ${ }^{[29]}$ When the reaction of $\mathbf{4 c}$ with $N$-methylmaleimide was performed under the optimized conditions

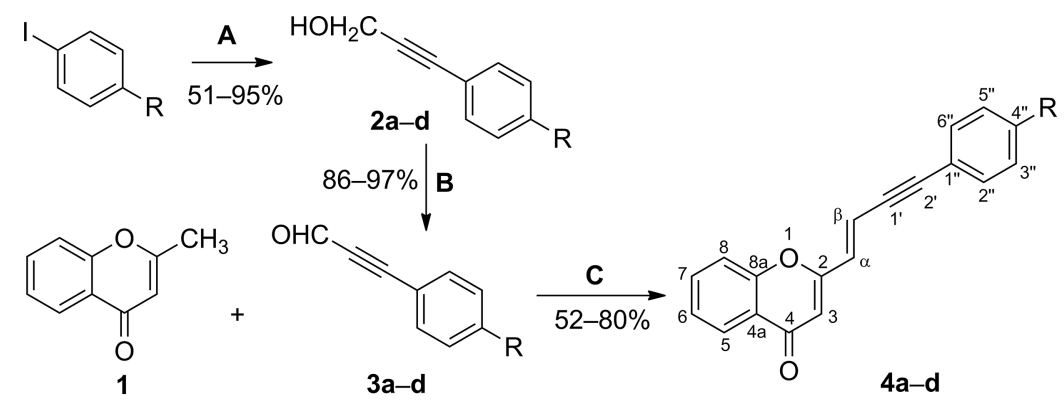

A: Toluene, $\mathrm{Pd}\left(\mathrm{PPh}_{3}\right)_{2} \mathrm{Cl}_{2}$, piperidine, Cul, propargyl alcohol, $60^{\circ} \mathrm{C}, 2 \mathrm{~h}$;

B: Ethyl acetate, $\mathrm{MnO}_{2}$, reflux, $1 \mathrm{~h}$;

C: $\mathrm{Na}, \mathrm{EtOH}$, r.t.

a: $\mathrm{R}=\mathrm{H} ; \mathbf{b}: \mathrm{R}=\mathrm{CH}_{3} ; \mathbf{c}: \mathrm{R}=\mathrm{OCH}_{3} ; \mathbf{d}: \mathrm{R}=\mathrm{Br}$

Scheme 1. Synthesis of (E)-2-(4-arylbut-1-en-3-yn-1-yl)chromones 4a-d. 


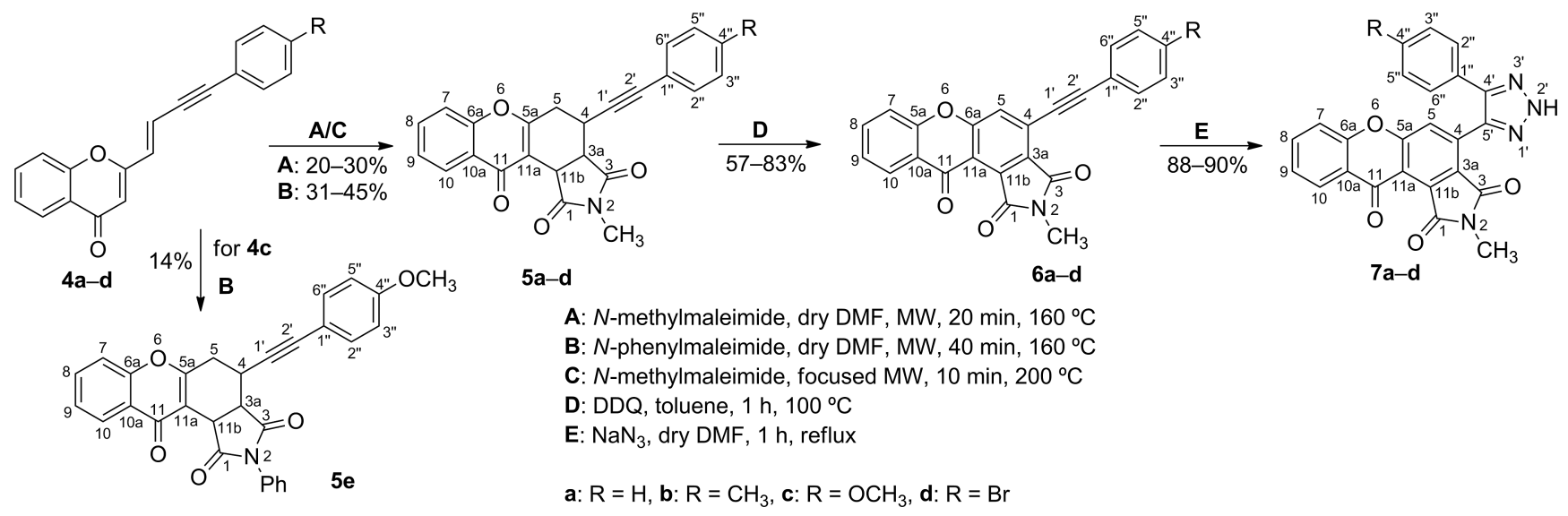

Scheme 2. Strategy for the synthesis of xanthone-1,2,3-triazole dyads $7 \mathbf{a}-\mathbf{d}$.

Table 1. Optimization of the Diels-Alder reaction conditions for the synthesis of 4-(arylethynyl)-2-methyl/phenyl-3a,4,5,11b-tetrahydrochromeno[3,2-e]isoindole-1,3,11-triones 5a-d.

\begin{tabular}{|c|c|c|c|c|c|c|c|c|}
\hline Entry & Heating method & Reactant & Solvent & Time & $T\left[{ }^{\circ} \mathrm{C}\right]$ & Catalyst & Isolated yield [\%] & Recovered starting material [\%] \\
\hline 1 & oil bath & $4 a$ & $1,2,4-\mathrm{TCB}$ & $7 \mathrm{~d}$ & reflux & - & no reaction & - \\
\hline 2 & oil bath & $4 c$ & $1,2,4-\mathrm{TCB}$ & $7 \mathrm{~d}$ & reflux & - & no reaction & - \\
\hline 3 & oil bath & $4 c$ & DMF & $48 \mathrm{~h}$ & reflux & $\mathrm{Sc}(\mathrm{OTf})_{3}$ & no reaction & - \\
\hline 4 & oil bath & $4 c$ & DMF & $48 \mathrm{~h}$ & reflux & - & no reaction & - \\
\hline 5 & oil bath & $4 b$ & [e] & $24 \mathrm{~h}$ & 80 & - & no reaction & - \\
\hline 6 & MW $(800 \mathrm{~W})$ & $4 c$ & $\mathrm{DMF}^{[\mathrm{a}]}$ & $40 \mathrm{~min}$ & 160 & - & 30 & 67 \\
\hline 7 & MW (800 W) & $4 c$ & $\mathrm{DMF}^{[\mathrm{a}]}$ & $40 \mathrm{~min}$ & 160 & $\mathrm{AlCl}_{3}$ & 30 & 65 \\
\hline 8 & MW (800 W) & $4 c$ & $\mathrm{DMF}^{[\mathrm{a}]}$ & $40 \mathrm{~min}$ & 160 & $\mathrm{Sc}(\mathrm{OTf})_{3}$ & 30 & 66 \\
\hline 9 & MW (800 W) & $4 c$ & $\mathrm{DMF}^{[\mathrm{a}]}$ & $20 \mathrm{~min}$ & 160 & - & 30 & 68 \\
\hline 10 & MW (800 W) & $4 c$ & $\mathrm{DMF}^{[\mathrm{a}]}$ & $10 \mathrm{~min}$ & 160 & - & 14 & 76 \\
\hline 11 & MW (800 W) & $4 c$ & $\mathrm{DMF}^{[\mathrm{c}]}$ & $40 \mathrm{~min}$ & 160 & - & no reaction & - \\
\hline 12 & MW (800 W) & $4 c$ & $\mathrm{NMP}^{[\mathrm{a}]}$ & $40 \mathrm{~min}$ & 160 & - & no reaction & - \\
\hline 13 & $\mathrm{MW}(600 \mathrm{~W})$ & $4 c$ & $\mathrm{NMP}^{[\mathrm{a}]}$ & $40 \mathrm{~min}$ & 150 & - & 5 & 70 \\
\hline 14 & MW (600 W) & $4 c$ & $\mathrm{NMP}^{[\mathrm{b}]}$ & $40 \mathrm{~min}$ & 150 & - & no reaction & - \\
\hline 15 & MW (600 W) & $4 c$ & $\mathrm{DMF}^{[\mathrm{b}]}$ & $40 \mathrm{~min}$ & 150 & - & no reaction & - \\
\hline 16 & MW (800 W) & $4 a$ & $\mathrm{DMF}^{[\mathrm{a}]}$ & $20 \mathrm{~min}$ & 160 & - & 28 & 67 \\
\hline 17 & MW (800 W) & $4 b$ & $\mathrm{DMF}^{[\mathrm{a}]}$ & $20 \mathrm{~min}$ & 160 & - & 20 & 72 \\
\hline 18 & MW (800 W) & $4 d$ & $\mathrm{DMF}^{[\mathrm{a}]}$ & $20 \mathrm{~min}$ & 160 & - & 30 & 66 \\
\hline 19 & MW (800 W) & $4 b$ & $\mathrm{DMF}^{[\mathrm{d}]}$ & $40 \mathrm{~min}$ & 160 & - & 14 & 10 \\
\hline
\end{tabular}

[a] A few drops of DMF and 5 equiv. of $N$-methylmaleimide. [b] $5 \mathrm{~mL}$ of solvent and 10 equiv. $N$-methylmaleimide. [c] Dimethyl acetylenedicarboxylate. [d] $N$-phenylmaleimide. [e] Dihydropyran.

$\left(160^{\circ} \mathrm{C}\right.$, using a few drops of DMF as solvent) for $10 \mathrm{~min}$ cycloadduct $\mathbf{5 c}$ was isolated in $14 \%$ yield; reaction times of 20 and 40 min afforded the same yield (30\%) (Table 1, Entries 6, 9 and 10). At lower reaction temperature $\left(100{ }^{\circ} \mathrm{C}\right)$, cycloadduct $\mathbf{5} \mathbf{c}$ was obtained in $1 \%$ yield and $80 \%$ of the starting material was recovered.

A single attempt to test $N$-phenylmaleimide as the dienophile led to no improvements in the DA reaction and led to only a $14 \%$ yield of cycloadduct $\mathbf{5 e}$ and $10 \%$ recovered starting material (Table 1, Entry 19, Scheme 2). The DA reaction has also been studied using monomode microwave equipment in solvent-free conditions. As an example, chromone $4 \mathbf{c}$ was used in three experiments performed at 5 , 10 and $20 \mathrm{~min}$ of irradiation at $200^{\circ} \mathrm{C}$. The best results (31\%) were not significantly better than those obtained under the multimode microwave conditions $(30 \%)$. However, it was possible to achieve similar results in shorter reaction times $(10 \mathrm{~min})$. Thus, after $5 \mathrm{~min}$ of irradiation it was pos- sible to isolate $\mathbf{5 c}$ in $23 \%$ yield; with 10 or 20 min of irradiation the yields increased to $31 \%$. Applying these optimized conditions to the other derivatives, we can conclude that the yields were slightly improved (36-45\%) using this environmentally friendlier technique since the reaction occurs in solvent-free conditions and in a shorter reaction time.

The next step of our first strategy was the oxidation/ aromatization of the DA adducts to afford xanthone derivatives. The reaction was performed in toluene with DDQ and the corresponding xanthone derivatives $\mathbf{6 a}-\mathbf{d}$ were achieved in good yields (Scheme 2, 57-83\%). The synthesized xanthones possess alkyne substitution in position 4 of the heterocycle, which, upon reacting with sodium azide in refluxing DMF provided new xanthone-1,2,3-triazole dyads 7a-d in excellent yields (Scheme 2, 88-90\%).

In the second strategy an inverse approach was used, starting with the synthesis of the triazole ring and then the xanthone skeleton. First, the 1,3-cycloaddition reaction of 

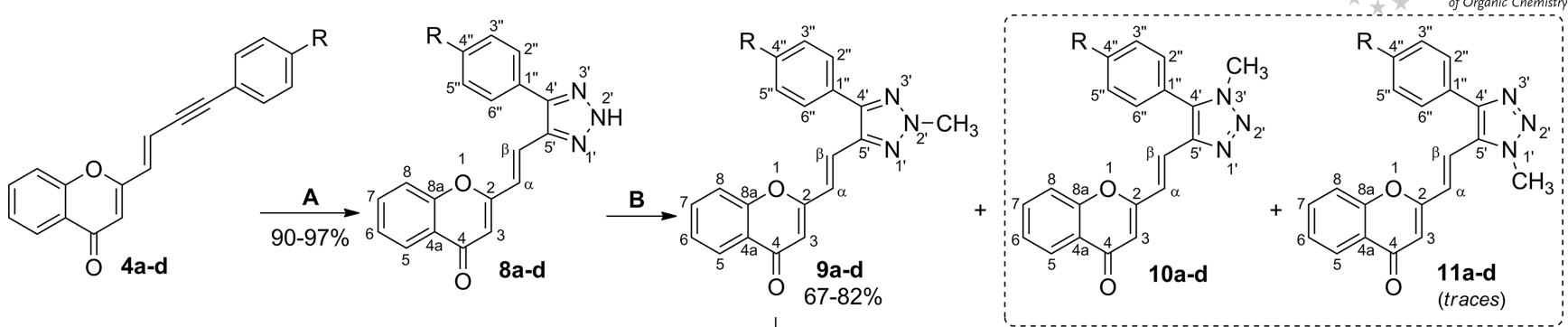

A: $\mathrm{NaN}_{3}$, dry DMF, $1 \mathrm{~h}$, reflux;

B: $\mathrm{K}_{2} \mathrm{CO}_{3}, \mathrm{Me}_{2} \mathrm{SO}_{4}$, acetone, $1 \mathrm{~h}$, reflux;

C: $N$-methylmaleimide, dry DMF, MW, $20 \mathrm{~min}, 160^{\circ} \mathrm{C}$;

D: $N$-methylmaleimide, focused $\mathrm{MW}, 10 \mathrm{~min}, 200^{\circ} \mathrm{C}$;

E: DDQ, toluene, $1 \mathrm{~h}, 100^{\circ} \mathrm{C}$.

a) $\mathrm{R}=\mathrm{H}$, b) $\mathrm{R}=\mathrm{CH}_{3}$, c) $\mathrm{R}=\mathrm{OCH}_{3}$, d) $\mathrm{R}=\mathrm{Br}$
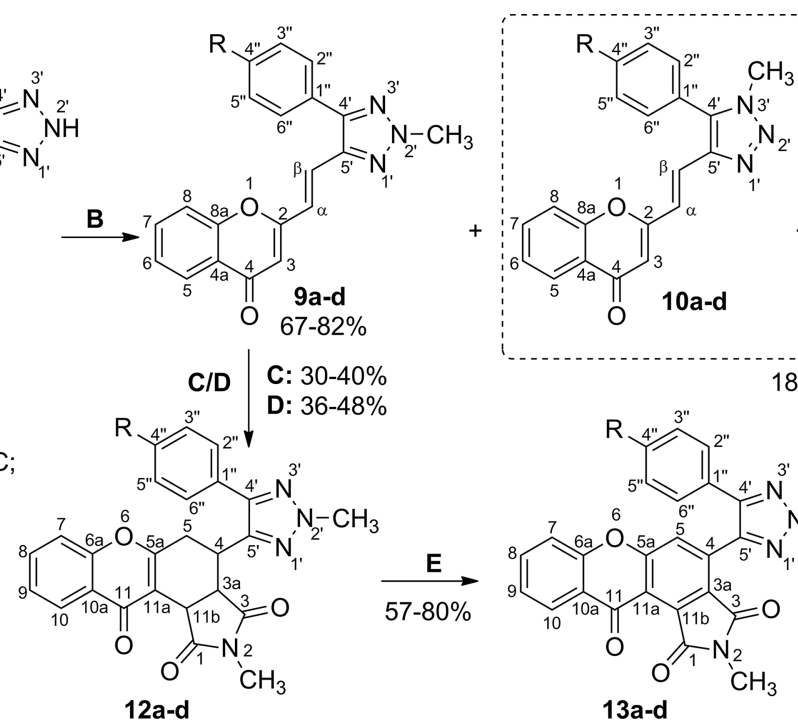

$18-33 \%$

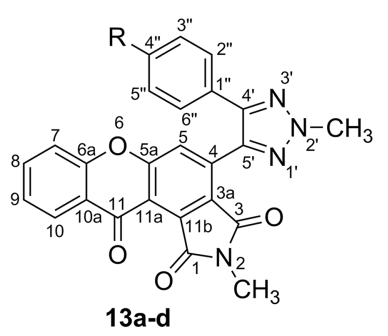

Scheme 3. Strategy for the synthesis of xanthone-1,2,3-triazole dyads 13a-d.

chromones 4a-d with sodium azide afforded the 1,2,3-triazole derivatives 8a-d in excellent yields (Scheme 3, 90$97 \%$ ). Subsequently and before the optimization of the DA reaction we protected the $\mathrm{NH}$ of the triazole since it could undergo a Michael addition reaction with the $N$-methylmaleimide dienophile. ${ }^{[30]} \mathrm{N}$-Protection with the methyl group was achieved using dimethyl sulfate in refluxing acetone and the expected three isomers of 1,2,3-triazoles $\mathbf{9 a}$ d, 10a-d and 11a-d were prepared in good overall yields (Scheme 3): $2^{\prime}-\mathrm{NCH}_{3}$ triazoles $9 \mathrm{a}-\mathbf{d}$ (higher $R_{\mathrm{f}}$ value) were isolated as major isomers $(67-82 \%)$; whereas $3^{\prime}-\mathrm{NCH}_{3}$ triazoles 10a-d together with $1^{\prime}-\mathrm{NCH}_{3}$ isomers $11 \mathbf{a}-\mathbf{d}$ were obtained as an inseparable mixture in low yields (18-33\%). Analysis of the NMR spectra of this mixture allowed us to conclude that $1^{\prime}-\mathrm{NCH}_{3}$ isomers 11a-d were obtained in trace amounts (see NMR discussion).

At this point and being aware that the DA reaction of chromone 4a-d only occurs under microwave irradiation conditions, the reaction of protected 1,2,3-triazole derivatives 9a-d with $N$-methylmaleimide was optimized using similar heating conditions. Once more, the lack of reactivity of the diene resulted in moderate yields of cycloadducts 12a-d $(30-40 \%)$ and $50-64 \%$ of recovered 9a-d when the reactions were performed in DMF at $160^{\circ} \mathrm{C}$ for $20 \mathrm{~min}$, with microwave irradiation (multimode apparatus). The optimization of the reaction using a monomode microwave apparatus was carried out using 1,2,3-triazole derivative 9c, in solvent-free conditions, with irradiation times of 5,10 and $20 \mathrm{~min}$ at $200^{\circ} \mathrm{C}$. The best yield of compound $12 \mathrm{c}$ was obtained with a $10 \mathrm{~min}$ irradiation; when applying these conditions to the other derivatives, the yields of cycloadducts 12a-d (36-48\%) were slightly better than those obtained using multimode microwave equipment. Furthermore, no side products were detected and $37-51 \%$ of starting materials 9a-d were recovered from the reaction mixtures. Finally, dehydrogenation of cycloadducts 12a-d lead- ing to xanthones 13a-d occurred in the presence of DDQ oxidant and with good results (Scheme 3, 57-80\%).

\section{Nuclear Magnetic Resonance Spectroscopy}

The most important features in the ${ }^{1} \mathrm{H}$ NMR spectra of the $(E)$-2-(4-arylbut-1-en-3-yn-1-yl)chromones $\mathbf{4 a - d}$ are, in each case: i) two doublets, at $\delta=6.66-6.71$ and 6.89$6.93 \mathrm{ppm}$ corresponding to the vinylic protons $\alpha-\mathrm{H}$ and $\beta$ $\mathrm{H}$ respectively, in a trans configuration $\left(J_{\alpha \mathrm{H}, \beta \mathrm{H}} 15-16 \mathrm{~Hz}\right)$, and ii) a singlet at $\delta=6.26-6.28 \mathrm{ppm}$ corresponding to $3-\mathrm{H}$. HMBC spectra enabled the assignment of the most relevant quaternary carbons: $\mathrm{C}-1^{\prime}$ and $\mathrm{C}-2^{\prime}$ at $\delta=86.7-88.4$ and 96.5-98.4 ppm, respectively, C-2 at $\delta=160.0-160.5 \mathrm{ppm}, \mathrm{C}-$ $8 \mathrm{a}$ and $\mathrm{C}-4 \mathrm{a}$ at $\delta=155.9$ and $124.1 \mathrm{ppm}$, respectively, and C-4 at $\delta=178.3 \mathrm{ppm}$ (Figure 1). In the NOESY spectra of compounds $\mathbf{4 a - d}$ NOE cross-peaks were observed between the 3-H signal and those of $\alpha-\mathrm{H}$ and $\beta-\mathrm{H}$ indicating free rotation around the $\mathrm{C} 2-\mathrm{C} \alpha$ bond.

The ${ }^{1} \mathrm{H}$ NMR spectra of DA cycloadducts $\mathbf{5 a - d}$, revealed the disappearance of the structural features mentioned for 4a-d. Furthermore, the ${ }^{1} \mathrm{H}$ NMR spectra showed the presence of five new signals: i) two multiplets at $\delta=3.02$ $3.08 \mathrm{ppm}$ and $\delta=3.70-3.77 \mathrm{ppm}$, corresponding to $5-\mathrm{H}$ and $4-\mathrm{H}$; ii) a double doublet at $\delta=3.28-3.29 \mathrm{ppm}$, corresponding to $3 \mathrm{a}-\mathrm{H}$; iii) a doublet at $\delta=4.37-4.39 \mathrm{ppm}$, corresponding to $11 \mathrm{~b}-\mathrm{H}$; and iv) a singlet at $\delta=2.96$ $2.98 \mathrm{ppm}$, corresponding to the $2-\mathrm{NCH}_{3}$ belonging to $\mathrm{N}$ methylmaleimide moiety. All these structural features of compounds 5a-d support the disappearance of structural features characteristic of compounds $\mathbf{4 a - d}$ indicating that the DA reaction proceeded thereby affording desired cycloadducts 5a-d. Analysis of the ${ }^{13} \mathrm{C}$ NMR spectra enabled assignment of three carbonyl carbons: $\mathrm{C}-11$ from the chromone moiety, and C-1 and C-3 corresponding to $N$-methyl- 

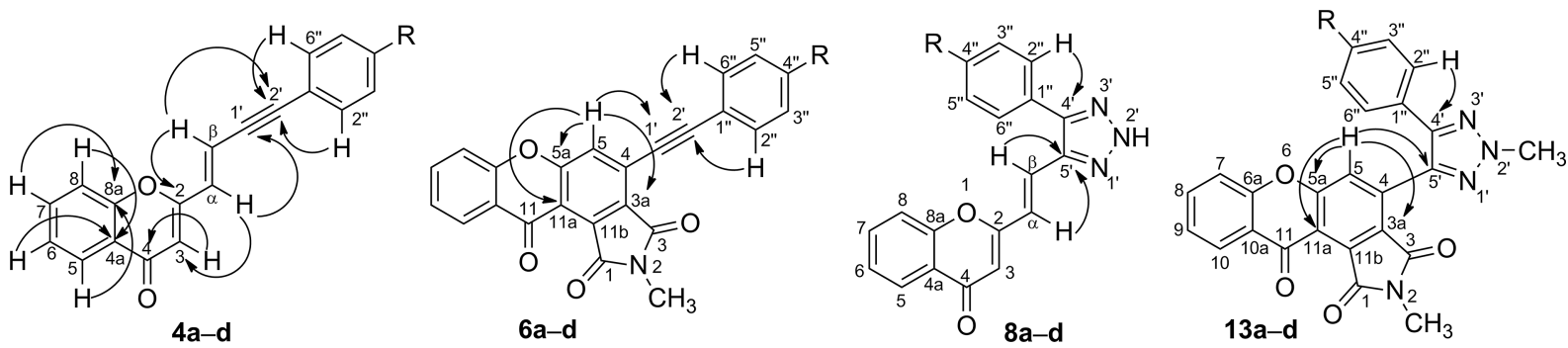

Figure 1. Important connectivities found in the HMBC spectra of the compounds $4 \mathbf{a}-\mathbf{d}, \mathbf{6 a}-\mathbf{d}, \mathbf{8 a}-\mathbf{d}$ and $\mathbf{1 3 a}-\mathbf{d}$.

maleimide. Furthermore, the HMBC spectra of derivatives 5a-d, allowed the assignment of carbons C-1' $(\delta=83.3-$ $84.8 \mathrm{ppm})$ and $\mathrm{C}-2^{\prime}(\delta=85.7-86.0 \mathrm{ppm})$ through the connectivity with aliphatic protons and with $\mathrm{H}-2^{\prime \prime}, 6^{\prime \prime}$, respectively.

The most important characteristics in the ${ }^{1} \mathrm{H}$ NMR spectra of 4-(arylethynyl)-2-methylchromeno[3,2-e]isoindole$1,3,11(2 H)$-triones $\mathbf{6 a}-\mathbf{d}$ are the presence in each case of two singlets, at $\delta=7.85-7.89 \mathrm{ppm}$ corresponding to the aromatic protons $5-\mathrm{H}$, and at $\delta=3.26 \mathrm{ppm}$ corresponding to the protons of the 2- $\mathrm{NCH}_{3}$ group of the $\mathrm{N}$-methylmaleimide moiety. Also, the aliphatic protons characteristic of DA adducts 5a-d do not appear in the aliphatic regions of the spectra.

The most relevant structural differences between compounds $6 \mathbf{a}-\mathbf{d}$ and $7 \mathbf{a}-\mathbf{d}$ are found in the ${ }^{13} \mathrm{C}$ NMR spectra. In compounds $\mathbf{6 a}-\mathbf{d}$ the signals corresponding to $\mathrm{C}-1^{\prime}$ and $\mathrm{C}-2^{\prime}$, assigned through HMBC analysis, appear at $\delta=83.4$ 85.0 and $99.0-101.2 \mathrm{ppm}$, respectively. These signals are not present in the ${ }^{13} \mathrm{C}$ NMR spectra of compounds $7 \mathbf{a}-\mathbf{d}$; instead, analysis of their ${ }^{13} \mathrm{C}$ NMR and HMBC spectra showed the presence of two signals at $\delta=136.3-139.3 \mathrm{ppm}$ and $\delta=142.1-142.7 \mathrm{ppm}$, corresponding to C-5' and C-4', respectively, of the 1,2,3-triazole moiety.

The multiplicity of the ${ }^{1} \mathrm{H}$ NMR spectra of the (E)-2-[2(4-aryl-2H-1,2,3-triazol-5-yl)vinyl]chromones 8a-d is pretty much the same as in (E)-2-(4-arylbut-1-en-3-yn-1-yl)chromones 4a-d. However, the signals of $\alpha-\mathrm{H}, \beta-\mathrm{H}$ and $3-\mathrm{H}(\delta=$ $7.30-7.35,7.61-7.68$ and $6.58-6.61 \mathrm{ppm}$, respectively) appear at higher frequency than those of the same protons in compounds $\mathbf{4 a - d}$. The deshielding effect of the triazole moiety is more pronounced for $\alpha-\mathrm{H}$ and $\beta-\mathrm{H}$ than for the 3$\mathrm{H}$ protons. As in the case of $\mathbf{4 a - d}$, the NOESY spectra of compounds 8a-d present NOE cross-peaks between the signal of $3-\mathrm{H}$ and those of $\alpha-\mathrm{H}$ and $\beta-\mathrm{H}$ indicating free rotation around the $\mathrm{C} 2-\mathrm{C} \alpha$ bond. In the case of the ${ }^{13} \mathrm{C}$ NMR spectra of compounds $\mathbf{8 a - d}$, the most important feature is the set of signals for $\mathrm{C}^{-} 4^{\prime}$ and $\mathrm{C}-5^{\prime}$, from 1,2,3-triazole moiety, at $\delta=137.6-138.3$ and $141.8-145.1 \mathrm{ppm}$, respectively, assigned from HMBC spectra (Figure 1).

In the ${ }^{1} \mathrm{H}$ NMR spectra of $(E)$-2-[2-(4-aryl-2-methyl-2H1,2,3-triazol-5-yl)vinyl]chromones $\mathbf{9 a}-\mathbf{d}$ the most relevant feature is the singlet at $\delta=4.28-4.29 \mathrm{ppm}$ corresponding to $2^{\prime}-\mathrm{NCH}_{3}$ of the methylated 1,2,3-triazole moiety. The unequivocal identification of isomers $9 \mathbf{a}-\mathbf{d}$ and $\mathbf{1 0 a}-\mathbf{d}$ was based on the connectivity found in the HMBC spectra. Thus, $2^{\prime}-\mathrm{NCH}_{3}$ protons of isomers $\mathbf{9 a}-\mathbf{d}$ do not correlate with any carbon of the 1,2,3-triazole ring. The position of the methyl group in the triazole ring of isomers $\mathbf{1 0 a}-\mathbf{d}$ was identified by correlations observed between $2^{\prime \prime}, 6^{\prime \prime}-\mathrm{H}$ and $3^{\prime}-\mathrm{NCH}_{3}$ with C-4' of the 1,2,3-triazole ring. Traces of isomers 11a-d were detected by the presence of less intense signals of the $1^{\prime}-\mathrm{NCH}_{3}$ protons and $3-\mathrm{H}$ in the ${ }^{1} \mathrm{H}$ NMR spectra of the mixture containing both 10a-d and 11a-d isomers.

Once again, the ${ }^{1} \mathrm{H}$ NMR spectra of the 4-(4-aryl-2methyl-2H-1,2,3-triazol-5-yl)-2-methyl-3a,4,5,11b-tetrahydrochromeno[3,2-e] isoindole-1,3,11(2H)-triones 12a-d do not show the structural features described for compounds $\mathbf{8 a}-\mathbf{d}$ and $\mathbf{9 a}-\mathbf{d}$. Indeed, new signals are present in these spectra: i) two multiplets at $\delta=2.96-3.24$ and 3.79 $3.91 \mathrm{ppm}$, corresponding to $5-\mathrm{H}$ and $4-\mathrm{H}$; ii) a double duplet at $\delta=3.46-3.48 \mathrm{ppm}$, due to $3 \mathrm{a}-\mathrm{H}$; iii) a doublet at $\delta$ $=4.63-4.64 \mathrm{ppm}$, assigned to $11 \mathrm{~b}-\mathrm{H}$; and iv) a singlet at $\delta$ $=2.81-2.82 \mathrm{ppm}$, attributed to $2-\mathrm{NCH}_{3}$ of the $\mathrm{N}$-methylmaleimide moiety. The key feature in the ${ }^{1} \mathrm{H}$ NMR spectra of 4-(5-aryl-2-methyl-2H-1,2,3-triazol-4-yl)-2-methylchromeno[3,2-e]isoindole-1,3,11(2H)-triones 13a-d is that of a singlet at $\delta=7.83-7.85 \mathrm{ppm}$ assigned to the 5-H aromatic proton in each case. Moreover, the ${ }^{1} \mathrm{H}$ NMR spectra of compounds 13a-d do not show aliphatic protons indicating successful aromatization of DA cycloadducts 12a-d.

\section{Conclusions}

We have reported the preparation of novel $(E)$-2-(4-arylbut-1-en-3-yn-1-yl)chromone derivatives, which were further used as starting materials in two main synthetic routes to xanthone-1,2,3-triazole dyads. The first strategy involves the Diels-Alder reaction of the chromone derivatives with $\mathrm{N}$-methylmaleimide followed by aromatization of the cycloadducts and reaction with sodium azide to give desired xanthone-1,2,3-triazole dyads. In the second synthetic approach an additional step of 1,2,3-triazole protection is required. Thus, chromone derivatives first react with sodium azide to provide chromone-1,2,3-triazole dyads, in excellent yields. After methylation of the triazole NH group, DA reaction with $N$-methylmaleimide and subsequent oxidation with DDQ affords the corresponding cycloadducts.

The DA reactions were performed under both monomode and multimode microwave irradiation conditions. The former case appears to be a more sustainable and environmentally friendly process since the reaction occurs in sol- 
vent-free conditions, a shorter reaction time and consequently less energy is required. Moreover, yields obtained in this "greener" fashion were slightly improved relative to those obtained from the multimode microwave irradiation conditions.

\section{Experimental Section}

General Remarks: Melting points were measured with a Büchi Melting Point B-540 apparatus. NMR spectra were recorded with a Bruker Avance 300 spectrometer $\left(300.13 \mathrm{MHz}\right.$ for ${ }^{1} \mathrm{H}$ and $75.47 \mathrm{MHz}$ for ${ }^{13} \mathrm{C}$ ) or Bruker Avance 500 spectrometer (500.13 MHz for ${ }^{1} \mathrm{H}$ and $125.77 \mathrm{MHz}$ for ${ }^{13} \mathrm{C}$ ). Chemical shifts $(\delta)$ are reported in ppm and coupling constants $(J)$ in $\mathrm{Hz}$; the internal standard was TMS. Unequivocal ${ }^{13} \mathrm{C}$ assignments were made with the aid of 2D $g \mathrm{HSQC}$ and $g \mathrm{HMBC}$ (delays for one-bond and longrange $J \mathrm{C} / \mathrm{H}$ couplings were optimised for 145 and $7 \mathrm{~Hz}$, respectively) experiments. Positive-ion ESI mass spectra were acquired with a QTOF 2 instrument [dilution of $1 \mu \mathrm{L}$ of the sample in chloroform solution (ca. $10^{-5} \mathrm{M}$ ) in $200 \mu \mathrm{L}$ of $0.1 \%$ trifluoroacetic acid/methanol solution. Nitrogen was used as nebuliser gas and argon as collision gas. The needle voltage was set at $3000 \mathrm{~V}$, with the ion source at $80^{\circ} \mathrm{C}$ and the desolvation temperature at $150{ }^{\circ} \mathrm{C}$. The cone voltage was $35 \mathrm{~V}$ ]. Other low- and high-resolution mass spectra (EI, $70 \mathrm{eV}$ ) were measured with VG Autospec Q and M spectrometers. Elemental analyses were obtained with a LECO 932 CHNS analyser. Preparative thin-layer chromatography was performed with Merck silica gel (60 DGF254). Column chromatography was performed with Merck silica gel (60, 70-230 mesh). All chemicals and solvents used were obtained from commercial sources and used as received or dried by standard procedures. 3Phenylpropiolaldehyde (3a) was not prepared since it is commercially available (Sigma-Aldrich).

General Procedure for the Synthesis of Arylpropargyl Alcohols 2bd: Propargyl alcohol $(1.75 \mathrm{~mL}, 30 \mathrm{mmol})$ was added to a solution of the appropriate iodobenzene (20 mmol), bis(triphenylphosphine) palladium(II) chloride $(280 \mathrm{mg}, 0.04 \mathrm{mmol})$, piperidine $(3.96 \mathrm{~mL}$, $40 \mathrm{mmol})$ and copper iodide $(114 \mathrm{mg}, 0.6 \mathrm{mmol})$ in toluene $(10 \mathrm{~mL})$, under a nitrogen atmosphere. The mixture was stirred at $60{ }^{\circ} \mathrm{C}$ for $2 \mathrm{~h}$ and then filtered through Celite 345 and washed with chloroform. The solvent was evaporated and the residue was purified by silica gel column chromatography using $\mathrm{CH}_{2} \mathrm{Cl}_{2}$ as eluent. The solvent was evaporated to dryness in each case, and expected 3-arylprop-2-yn-1-ols 2b-d were obtained in good yields (79-95\%).

3-(4-Methylphenyl)prop-2-yn-1-ol (2b): Yield $2.31 \mathrm{~g}(79 \%)$. Oil. ${ }^{1} \mathrm{H}$ NMR (300 MHz, $\left.\mathrm{CDCl}_{3}\right): \delta=2.34\left(\mathrm{~s}, 3 \mathrm{H}, 4^{\prime}-\mathrm{CH}_{3}\right), 4.48$ (d, $J=$ $5.2 \mathrm{~Hz}, 2 \mathrm{H}, 1-\mathrm{H}), 7.11\left(\mathrm{~d}, J=7.9 \mathrm{~Hz}, 2 \mathrm{H}, 3^{\prime}, 5^{\prime}-\mathrm{H}\right), 7.33$ (d, $J=$ $\left.7.9 \mathrm{~Hz}, 2 \mathrm{H}, 2^{\prime}, 6^{\prime}-\mathrm{H}\right) \mathrm{ppm} .{ }^{13} \mathrm{C} \mathrm{NMR}\left(75 \mathrm{MHz}, \mathrm{CDCl}_{3}\right): \delta=21.4$ $\left(4^{\prime}-\mathrm{CH}_{3}\right), 51.6(\mathrm{C}-1), 85.7(\mathrm{C}-3), 86.5(\mathrm{C}-2), 119.3\left(\mathrm{C}-1^{\prime}\right), 129.0(\mathrm{C}-$ $\left.3^{\prime}, 5^{\prime}\right), 131.5\left(\mathrm{C}-2^{\prime}, 6^{\prime}\right), 138.6\left(\mathrm{C}-4^{\prime}\right) \mathrm{ppm}$. HRMS $\left(\mathrm{ESI}^{+}\right): \mathrm{m} / z$ calcd. for $\mathrm{C}_{10} \mathrm{H}_{11} \mathrm{O}[\mathrm{M}+\mathrm{H}]^{+}$147.0810, found 147.0801. $\mathrm{MS}\left(\mathrm{ESI}^{+}\right): \mathrm{m} / z$ $(\%)=147(100)[\mathrm{M}+\mathrm{H}]^{+}, 315(35)[2 \mathrm{M}+\mathrm{Na}]^{+}$.

3-(4-Methoxyphenyl)prop-2-yn-1-ol (2c): Yield $2.56 \mathrm{~g}$ (79\%). This compound showed spectroscopic and analytical data identical to previously reported data. ${ }^{[31]}$

3-(4-Bromophenyl)prop-2-yn-1-ol (2d): Yield $4.01 \mathrm{~g}$ (95\%). This compound showed spectroscopic and analytical data identical to previously reported data. ${ }^{[32]}$

General Procedure for the Synthesis of Arylpropargyl Aldehydes 3bd: Activated $\mathrm{MnO}_{2}(6.9 \mathrm{~g}, 79.6 \mathrm{mmol})$ was added to a solution of the appropriate arylpropargyl alcohols $\mathbf{2 b - d}(15.9 \mathrm{mmol})$ in EtOAc
$(40 \mathrm{~mL})$. The resulting mixture was then refluxed for $1 \mathrm{~h}$. After that period, the mixture was filtered through Celite 345 and washed with EtOAc and $\mathrm{CH}_{2} \mathrm{Cl}_{2}$. After evaporation of the solvent, the residue was purified by silica gel column chromatography using $\mathrm{CH}_{2} \mathrm{Cl}_{2}$ as eluent. The solvent was evaporated to dryness to give the expected arylpropynals $\mathbf{3 b}-\mathbf{d}$ in good yields (86-97\%).

3-(4-Methylphenyl)propiolaldehyde (3b): Yield $1.97 \mathrm{~g}$ (86\%). Oil. ${ }^{1} \mathrm{H}$ NMR (300 MHz, $\left.\mathrm{CDCl}_{3}\right): \delta=2.40\left(\mathrm{~s}, 3 \mathrm{H}, 4^{\prime}-\mathrm{CH}_{3}\right), 7.22(\mathrm{~d}, J=$ $\left.8.0 \mathrm{~Hz}, 2 \mathrm{H}, 3^{\prime}, 5^{\prime}-\mathrm{H}\right), 7.51\left(\mathrm{~d}, J=8.0 \mathrm{~Hz}, 2 \mathrm{H}, 2^{\prime}, 6^{\prime}-\mathrm{H}\right), 9.41$ (s, 1 $\mathrm{H}, 1-\mathrm{H}) \mathrm{ppm} .{ }^{13} \mathrm{C} \mathrm{NMR}\left(300 \mathrm{MHz}, \mathrm{CDCl}_{3}\right): \delta=21.8\left(4^{\prime}-\mathrm{CH}_{3}\right)$, 88.4 (C-2), 96.0 (C-3), 116.3 (C-1'), 129.5 (C-3',5'), 133.3 (C-2', 6'), 142.2 (C-4'), 176.9 (C-1) ppm. HRMS (ESI $\left.{ }^{+}\right): m / z$ calcd. for $\mathrm{C}_{10} \mathrm{H}_{9} \mathrm{O}[\mathrm{M}+\mathrm{H}]^{+}$145.0653, found 145.0644. MS (ESI $\left.{ }^{+}\right): \mathrm{m} / z(\%)$ $=189(55)[\mathrm{M}+2 \mathrm{Na}]^{+}, 145(30)[\mathrm{M}+\mathrm{H}]^{+}$.

3-(4-Methoxyphenyl)propiolaldehyde (3c): Yield $2.34 \mathrm{~g}$ (92\%). This compound showed spectroscopic and analytical data identical to previously reported data. ${ }^{[31]}$

3-(4-Bromophenyl)propiolaldehyde (3d): Yield $3.22 \mathrm{~g}$ (97\%). This compound showed spectroscopic and analytical data identical to previously reported data. ${ }^{[32]}$

General Procedure for the Synthesis of 2-(4-Arylbut-1-en-3-yn-1-yl)4H-chromen-4-ones 4a-d: Sodium $(0.11 \mathrm{~g}$, $4.8 \mathrm{mmol})$ was gradually added to $5 \mathrm{~mL}$ of dry ethanol and the mixture was stirred until the reaction mixture reached room temperature. 2-Methylchromone 1 $(0.2 \mathrm{~g}, 1.2 \mathrm{mmol})$ and the appropriate aldehyde $\mathbf{3 a}-\mathbf{d}(1.8 \mathrm{mmol})$ were added and the reaction mixture allowed to stand at room temperature until complete disappearance of chromone 1. After this period, the solution was poured into ice $(20 \mathrm{~g})$ and water $(30 \mathrm{~mL})$ and the $\mathrm{pH}$ adjusted to 4 with dilute $\mathrm{HCl}$. The solid was removed by filtration, taken in $\mathrm{CH}_{2} \mathrm{Cl}_{2}$ and purified by silica gel column chromatography using $\mathrm{CH}_{2} \mathrm{Cl}_{2}$ as eluent. The solvent was evaporated to dryness and the residue were recrystallized from ethanol to give the (E)-2-(4-arylbut-1-en-3-yn-1-yl)-4H-chromen-4ones $4 \mathbf{a}-\mathbf{d}$ in good yields $(52-80 \%)$.

(E)-2-(4-Phenylbut-1-en-3-yn-1-yl)-4H-chromen-4-one (4a): Yield $166 \mathrm{mg}$ (52\%), m.p. $127-128^{\circ} \mathrm{C}$ (recrystallized from ethanol). ${ }^{1} \mathrm{H}$ NMR $\left(300 \mathrm{MHz}, \mathrm{CDCl}_{3}\right): \delta=6.28(\mathrm{~s}, 1 \mathrm{H}, 3-\mathrm{H}), 6.71(\mathrm{~d}, J=$ $15.7 \mathrm{~Hz}, 1 \mathrm{H}, \alpha-\mathrm{H}), 6.93(\mathrm{~d}, J=15.7 \mathrm{~Hz}, 1 \mathrm{H}, \beta-\mathrm{H}), 7.35-7.43(\mathrm{~m}$, $\left.4 \mathrm{H}, 6-\mathrm{H}, 3^{\prime \prime}, 4^{\prime \prime}, 5^{\prime \prime}-\mathrm{H}\right), 7.48$ (d, $\left.J=7.7 \mathrm{~Hz}, 1 \mathrm{H}, 8-\mathrm{H}\right), 7.48-7.52$ (m, $\left.2 \mathrm{H}, 2^{\prime \prime}, 6^{\prime \prime}-\mathrm{H}\right), 7.69$ (dt, $\left.J=7.7,1.7 \mathrm{~Hz}, 1 \mathrm{H}, 7-\mathrm{H}\right), 8.19$ (dd, $J=7.9,1.7 \mathrm{~Hz}, 1 \mathrm{H}, 5-\mathrm{H}) \mathrm{ppm} .{ }^{13} \mathrm{C} \mathrm{NMR}\left(75 \mathrm{MHz}, \mathrm{CDCl}_{3}\right): \delta=$ $87.4\left(\mathrm{C}-1^{\prime}\right), 97.8\left(\mathrm{C}-2^{\prime}\right), 111.1$ (C-3), $117.2(\mathrm{C}-\beta), 117.9$ (C-8), 122.4 $\left(\mathrm{C}-1^{\prime \prime}\right), 124.1$ (C-4a), 125.2 (C-6), 125.7 (C-5), $128.5\left(\mathrm{C}-3^{\prime \prime}, 5^{\prime \prime}\right)$, $129.2\left(\mathrm{C}-4^{\prime \prime}\right), 131.9\left(\mathrm{C}-2^{\prime \prime}, 6^{\prime \prime}\right), 132.1(\mathrm{C}-\alpha), 134.0$ (C-7), 155.9 (C8a), 160.2 (C-2), 178.3 (C-4) ppm. $\mathrm{C}_{19} \mathrm{H}_{12} \mathrm{O}_{2}$ : C, 83.81; H, 4.44; found $\mathrm{C}, 83.48 ; \mathrm{H}, 4.40 . \mathrm{MS}\left(\mathrm{ESI}^{+}\right): \mathrm{m} / z(\%)=273(100)[\mathrm{M}+$ $\mathrm{H}]^{+}, 295(54)[\mathrm{M}+\mathrm{Na}]^{+}$.

(E)-2-[4-(4-Methylphenyl)but-1-en-3-yn-1-yl]-4H-chromen-4-one (4b): Yield $286 \mathrm{mg}(80 \%)$, m.p. $148-150{ }^{\circ} \mathrm{C}$ (recrystallized from ethanol). ${ }^{1} \mathrm{H} \mathrm{NMR}\left(300 \mathrm{MHz}, \mathrm{CDCl}_{3}\right): \delta=2.38\left(\mathrm{~s}, 3 \mathrm{H}, 4^{\prime \prime}-\mathrm{CH}_{3}\right)$, 6.27 (s, $1 \mathrm{H}, 3-\mathrm{H}), 6.69$ (d, $J=15.8 \mathrm{~Hz}, 1 \mathrm{H}, \alpha-\mathrm{H}), 6.93$ (d, $J=$ $15.8 \mathrm{~Hz}, 1 \mathrm{H}, \beta-\mathrm{H}), 7.18\left(\mathrm{~d}, J=8.1 \mathrm{~Hz}, 2 \mathrm{H}, 3^{\prime \prime}, 5^{\prime \prime}-\mathrm{H}\right), 7.400$ (ddd, $J=7.6,7.5,1.0 \mathrm{~Hz}, 1 \mathrm{H}, 6-\mathrm{H}), 7.402\left(\mathrm{~d}, J=8.1 \mathrm{~Hz}, 2 \mathrm{H}, 2^{\prime \prime}, 6^{\prime \prime}\right.$ H), $7.48(\mathrm{dd}, J=7.9,1.0 \mathrm{~Hz}, 1 \mathrm{H}, 8-\mathrm{H}), 7.69$ (ddd, $J=7.9,7.6$, $1.7 \mathrm{~Hz}, 1 \mathrm{H}, 7-\mathrm{H}), 8.19$ (dd, $J=7.6,1.7 \mathrm{~Hz}, 1 \mathrm{H}, 5-\mathrm{H}) \mathrm{ppm} .{ }^{13} \mathrm{C}$ NMR (75 MHz, $\left.\mathrm{CDCl}_{3}\right): \delta=21.6\left(4^{\prime \prime}-\mathrm{CH}_{3}\right), 87.0\left(\mathrm{C}-1^{\prime \prime}\right), 98.3(\mathrm{C}-$ 2'), 111.0 (C-3), 117.4 (C- $\beta$ ), 117.9 (C-8), 119.3 (C-1'), 124.1 (C4a), 125.1 (C-6), 125.7 (C-5), $129.3\left(\mathrm{C}-3^{\prime \prime}, 5^{\prime \prime}\right), 131.6$ (C- $\left.\alpha\right), 131.8$ $\left(\mathrm{C}-2^{\prime \prime}, 6^{\prime \prime}\right), 133.9$ (C-7), 139.6 (C-4" ), 155.9 (C-8a), 160.4 (C-2), 178.3 (C-4) ppm. $\mathrm{C}_{20} \mathrm{H}_{14} \mathrm{O}_{2}: \mathrm{C}, 83.90 ; \mathrm{H}, 4.93$; found $\mathrm{C}, 83.51 ; \mathrm{H}$, 4.91. $\mathrm{MS}\left(\mathrm{ESI}^{+}\right): m / z(\%)=287(100)[\mathrm{M}+\mathrm{H}]^{+}, 325(12)[\mathrm{M}+$ $\mathrm{K}]^{+}, 595(8)[2 \mathrm{M}+\mathrm{Na}]^{+}$. 
(E)-2-[4-(4-Methoxyphenyl)but-1-en-3-yn-1-yl]-4H-chromen-4-one (4c): Yield $302 \mathrm{mg}(80 \%)$, m.p. $162-163{ }^{\circ} \mathrm{C}$ (recrystallized from ethanol). ${ }^{1} \mathrm{H}$ NMR (300 MHz, $\left.\mathrm{CDCl}_{3}\right): \delta=3.85$ (s, $3 \mathrm{H}, 4^{\prime \prime}-\mathrm{OCH}_{3}$ ), $6.26(\mathrm{~s}, 1 \mathrm{H}, 3-\mathrm{H}), 6.66(\mathrm{~d}, J=15.7 \mathrm{~Hz}, 1 \mathrm{H}, \alpha-\mathrm{H}), 6.89(\mathrm{~d}, J=$ $\left.8.6 \mathrm{~Hz}, 2 \mathrm{H}, 3^{\prime \prime}, 5^{\prime \prime}-\mathrm{H}\right), 6.93$ (d, $\left.J=15.7 \mathrm{~Hz}, 1 \mathrm{H}, \beta-\mathrm{H}\right), 7.40$ (dd, $J=7.9,7.8 \mathrm{~Hz}, 1 \mathrm{H}, 6-\mathrm{H}), 7.45\left(\mathrm{~d}, J=8.6 \mathrm{~Hz}, 2 \mathrm{H}, 2^{\prime \prime}, 6^{\prime \prime}-\mathrm{H}\right)$, $7.48(\mathrm{~d}, J=7.6 \mathrm{~Hz}, 1 \mathrm{H}, 8-\mathrm{H}), 7.69$ (ddd, $J=7.8,7.6,1.6 \mathrm{~Hz}, 1$ $\mathrm{H}, 7-\mathrm{H}), 8.19(\mathrm{dd}, J=7.9,1.6 \mathrm{~Hz}, 1 \mathrm{H}, 5-\mathrm{H}) \mathrm{ppm} .{ }^{13} \mathrm{C} \mathrm{NMR}$ $\left(75 \mathrm{MHz}, \mathrm{CDCl}_{3}\right): \delta=55.4\left(4^{\prime \prime}-\mathrm{OCH}_{3}\right), 86.7\left(\mathrm{C}-1^{\prime}\right), 98.4\left(\mathrm{C}-2^{\prime}\right)$,

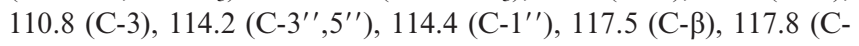
8), 124.1 (C-4a), 125.1 (C-6), 125.7 (C-5), 131.1 (C- $\alpha$ ), 133.5 (C$\left.2^{\prime \prime}, 6^{\prime \prime}\right), 133.9$ (C-7), 155.9 (C-8a), 160.4 (C-4"'), 160.5 (C-2), 178.3 (C-4) ppm. $\mathrm{C}_{20} \mathrm{H}_{14} \mathrm{O}_{3}$ : C, 79.46; H, 4.67; found $\mathrm{C}, 79.61 ; \mathrm{H}, 4.83$. MS $\left(\mathrm{ESI}^{+}\right): m / z(\%)=303(86)[\mathrm{M}+\mathrm{H}]^{+}, 325(53)[\mathrm{M}+\mathrm{Na}]^{+}, 627$ (100) $[2 \mathrm{M}+\mathrm{Na}]^{+}$.

(E)-2-[4-(4-Bromophenyl)but-1-en-3-yn-1-yl]-4H-chromen-4-one (4d): Yield $259 \mathrm{mg}(59 \%)$, m.p. $164-166^{\circ} \mathrm{C}$ (recrystallized from ethanol). ${ }^{1} \mathrm{H}$ NMR (300 MHz, $\left.\mathrm{CDCl}_{3}\right): \delta=6.28(\mathrm{~s}, 1 \mathrm{H}, 3-\mathrm{H}), 6.71$ $(\mathrm{d}, J=15.8 \mathrm{~Hz}, 1 \mathrm{H}, \alpha-\mathrm{H}), 6.89(\mathrm{~d}, J=15.8 \mathrm{~Hz}, 1 \mathrm{H}, \beta-\mathrm{H}), 7.36$ (d, $\left.J=8.6 \mathrm{~Hz}, 2 \mathrm{H}, 2^{\prime \prime}, 6^{\prime \prime}-\mathrm{H}\right), 7.40(\mathrm{dt}, J=7.8,0.9 \mathrm{~Hz}, 1 \mathrm{H}, 6-$ H), $7.48(\mathrm{dd}, J=8.7,0.9 \mathrm{~Hz}, 1 \mathrm{H}, 8-\mathrm{H}), 7.51(\mathrm{~d}, J=8.6 \mathrm{~Hz}, 2 \mathrm{H}$, $\left.3^{\prime \prime}, 5^{\prime \prime}-\mathrm{H}\right), 7.69$ (ddd, $\left.J=8.7,7.8,1.7 \mathrm{~Hz}, 1 \mathrm{H}, 7-\mathrm{H}\right), 8.19$ (dd, $J=$ 7.8, $1.7 \mathrm{~Hz}, 1 \mathrm{H}, 5-\mathrm{H}) \mathrm{ppm} .{ }^{13} \mathrm{C} \mathrm{NMR}\left(75 \mathrm{MHz}, \mathrm{CDCl}_{3}\right): \delta=88.4$ $\left(\mathrm{C}-1^{\prime}\right), 96.5\left(\mathrm{C}-2^{\prime}\right), 111.3$ (C-3), 116.7 (C- $\left.\beta\right), 117.8$ (C-8), 121.3 (C$\left.1^{\prime \prime}\right), 123.6\left(\mathrm{C}-4^{\prime \prime}\right), 124.1$ (C-4a), 125.2 (C-6), 125.7 (C-5), 131.8 (C$\left.3^{\prime \prime}, 5^{\prime \prime}\right), 132.5(\mathrm{C}-\alpha), 133.2\left(\mathrm{C}-2^{\prime \prime}, 6^{\prime \prime}\right), 134.0$ (C-7), 155.9 (C-8a), 160.0 (C-2), 178.3 (C-4) ppm. $\mathrm{C}_{19} \mathrm{H}_{11} \mathrm{BrO}_{2}$ : C, 64.98; H, 3.16; found $\mathrm{C}, 64.71 ; \mathrm{H}, 3.23$. MS $\left(\mathrm{ESI}^{+}\right): m / z(\%)=351(39)[\mathrm{M}+\mathrm{H}]^{+}\left({ }^{79} \mathrm{Br}\right)$; $353(37)[\mathrm{M}+\mathrm{H}]^{+}\left({ }^{81} \mathrm{Br}\right)$.

General Procedure for the Synthesis of 4-(Arylethynyl)-2-methyl3a,4,5,11b-tetrahydrochromeno[3,2-e]isoindole-1,3,11(2H)-triones 5a-e. Method A: $N$-Methylmaleimide $(0.10 \mathrm{~g}, 0.92 \mathrm{mmol})$ was added to a solution of the appropriate (E)-2-(4-arylbut-1-en-3-yn1-yl)-4H-chromen-4-one (4a-d) $(0.18 \mathrm{mmol})$ in dry DMF $(5 \mu \mathrm{L})$. The mixture was heated at $160{ }^{\circ} \mathrm{C}$ under microwave irradiation (multimode apparatus) for $20 \mathrm{~min}$. The residue was dissolved in $\mathrm{CH}_{2} \mathrm{Cl}_{2}$ and purified by preparative TLC using $\mathrm{CH}_{2} \mathrm{Cl}_{2}$ as eluent to give desired cycloadducts $\mathbf{5 a}-\mathbf{d}$ : $\mathbf{5 a}(19.7 \mathrm{mg}, 28 \%), \mathbf{5 b}(19.3 \mathrm{mg}$, $20 \%), 5$ c $(22.3 \mathrm{mg}, 30 \%), 5 d(25.0 \mathrm{mg}, 30 \%)$. Adduct 5e was obtained in $14 \%$ using the same methodology, under irradiation for $40 \mathrm{~min}$.

Method B: $N$-Methylmaleimide $(0.10 \mathrm{~g}, 0.92 \mathrm{mmol})$ was mixed with the appropriate $(E)-2$-(4-arylbut-1-en-3-yn-1-yl)-4H-chromen-4one $4 \mathbf{a}-\mathbf{d}(0.18 \mathrm{mmol})$ in a closed vessel. The mixture was heated at $200{ }^{\circ} \mathrm{C}$ under microwave irradiation (monomode apparatus) for $10 \mathrm{~min}$. The residue was dissolved in $\mathrm{CH}_{2} \mathrm{Cl}_{2}$ and purified by preparative TLC using $\mathrm{CH}_{2} \mathrm{Cl}_{2}$ as eluent to give desired cycloadducts 5a-d: 5a (25.3 mg, 36\%), 5b (40.5 mg, 42\%), 5c (23.0 mg, 31\%), 5d (37.5 mg, 45\%).

2-Methyl-4-(phenylethynyl)-3a,4,5,11b-tetrahydrochromeno[3,2-e]isoindole-1,3,11(2H)-trione (5a): M.p. $169-171^{\circ} \mathrm{C} .{ }^{1} \mathrm{H}$ NMR $\left(300 \mathrm{MHz}, \mathrm{CDCl}_{3}\right): \delta=2.98\left(\mathrm{~s}, 3 \mathrm{H}, \mathrm{CH}_{3}\right), 3.04-3.08(\mathrm{~m}, 2 \mathrm{H}, 5-$ H), 3.29 (dd, $J=7.6,5.0 \mathrm{~Hz}, 1 \mathrm{H}, 3 \mathrm{a}-\mathrm{H}), 3.73-3.77$ (m, $1 \mathrm{H}, 4-\mathrm{H})$, $4.39(\mathrm{~d}, J=7.6 \mathrm{~Hz}, 1 \mathrm{H}, 11 \mathrm{~b}-\mathrm{H}), 7.21-7.28(\mathrm{~m}, 5 \mathrm{H}$, $\left.2^{\prime \prime}, 3^{\prime \prime}, 4^{\prime \prime}, 5^{\prime \prime}, 6^{\prime \prime}-\mathrm{H}\right), 7.39-7.45$ (m, 2 H, 7-H, 9-H), 7.67 (ddd, $J=$ 8.6, 7.1, $1.7 \mathrm{~Hz}, 1 \mathrm{H}, 8-\mathrm{H}), 8.29$ (dd, $J=8.2,1.7 \mathrm{~Hz}, 1 \mathrm{H}, 10-$ H) ppm. ${ }^{13} \mathrm{C}$ NMR $\left(75 \mathrm{MHz}, \mathrm{CDCl}_{3}\right): \delta=24.8\left(\mathrm{NCH}_{3}\right), 27.8(\mathrm{C}-$ 4), 33.2 (C-5), 37.8 (C-11b), 43.2 (C-3a), 84.7 (C-1'), 85.8 (C-2'), 112.1 (C-11a), 117.6 (C-7), 121.7 (C-1' $), 123.5$ (C-10a), 125.2 (C9), $126.4(\mathrm{C}-10), 128.2\left(\mathrm{C}-2^{\prime \prime}, 6^{\prime \prime}\right), 128.6\left(\mathrm{C}-4^{\prime \prime}\right), 131.6\left(\mathrm{C}-3^{\prime \prime}, 5^{\prime \prime}\right)$, 133.7 (C-8), 155.7 (C-6a), 162.1 (C-5a), 174.2 (C-3 and C-11), 176.1 (C-1) ppm. HRMS $\left(\mathrm{ESI}^{+}\right): \mathrm{m} / z$ calcd. for $\mathrm{C}_{24} \mathrm{H}_{18} \mathrm{NO}_{4}[\mathrm{M}+\mathrm{H}]^{+}$
384.1236, found 384.1218. MS $\left(\mathrm{ESI}^{+}\right): \mathrm{m} / z(\%)=384(32)[\mathrm{M}+$ $\mathrm{H}]^{+}, 406(94)[\mathrm{M}+\mathrm{Na}]^{+}, 789(100)[2 \mathrm{M}+\mathrm{Na}]^{+}$.

2-Methyl-4-[(4-methylphenyl)ethynyl]-3a,4,5,11b-tetrahydrochromeno[3,2-e]isoindole-1,3,11(2H)-trione (5b): M.p. $197-199{ }^{\circ} \mathrm{C}$. ${ }^{1} \mathrm{H}$ NMR (300 MHz, $\left.\mathrm{CDCl}_{3}\right): \delta=2.28\left(\mathrm{~s}, 3 \mathrm{H}, 4^{\prime \prime}-\mathrm{CH}_{3}\right), 2.97$ (s, 3 $\left.\mathrm{H}, \mathrm{N}-\mathrm{CH}_{3}\right), 3.03-3.07(\mathrm{~m}, 1 \mathrm{H}, 5-\mathrm{H}), 3.28(\mathrm{dd}, J=7.6,5.0 \mathrm{~Hz}, 1$ H, 3a-H), 3.72-3.76 (m, $1 \mathrm{H}, 4-\mathrm{H}), 4.37$ (d, $J=7.6 \mathrm{~Hz}, 1 \mathrm{H}, 11 \mathrm{~b}-$ $\mathrm{H}), 7.01\left(\mathrm{~d}, J=7.1 \mathrm{~Hz}, 2 \mathrm{H}, 3^{\prime \prime}, 5^{\prime \prime}-\mathrm{H}\right), 7.16(\mathrm{~d}, J=7.1 \mathrm{~Hz}, 2 \mathrm{H}$, $\left.2^{\prime \prime}, 6^{\prime \prime}-\mathrm{H}\right), 7.39-7.44(\mathrm{~m}, 2 \mathrm{H}, 7-\mathrm{H}, 9-\mathrm{H}), 7.66(\mathrm{ddd}, J=8.5,7.1$, $1.7 \mathrm{~Hz}, 1 \mathrm{H}, 8-\mathrm{H}), 8.29$ (dd, $J=8.2,1.7 \mathrm{~Hz}, 1 \mathrm{H}, 10-\mathrm{H}) \mathrm{ppm} .{ }^{13} \mathrm{C}$ NMR $\left(75 \mathrm{MHz}, \mathrm{CDCl}_{3}\right): \delta=21.4\left(4^{\prime \prime}-\mathrm{CH}_{3}\right), 24.8\left(\mathrm{~N}-\mathrm{CH}_{3}\right), 27.8$ (C-4), 33.2 (C-5), 37.7 (C-11b), 43.3 (C-3a), 84.0 (C-1'), 85.9 (C2'), 112.1 (C-11a), 117.6 (C-9), 118.6 (C-1' ), 123.5 (C-10a), 125.2 (C-7), 126.4 (C-10), $129.0\left(\mathrm{C}-3^{\prime \prime}, 5^{\prime \prime}\right), 131.4\left(\mathrm{C}-2^{\prime \prime}, 6^{\prime \prime}\right), 133.7$ (C-8), 138.8 (C-4"'), 155.7 (C-6a), 162.2 (C-5a), 174.3 (C-3), 176.2 (C-1 and C-11) ppm. HRMS (ESI $\left.{ }^{+}\right): m / z$ calcd. for $\mathrm{C}_{25} \mathrm{H}_{20} \mathrm{NO}_{4}[\mathrm{M}+$ $\mathrm{H}]^{+}$398.1392, found 398.1372. MS $\left(\mathrm{ESI}^{+}\right): m / z(\%)=398(100)[\mathrm{M}$ $+\mathrm{H}]^{+}, 420(21)[\mathrm{M}+\mathrm{Na}]^{+}, 817(27)[2 \mathrm{M}+\mathrm{Na}]^{+}$.

4-[(4-Methoxyphenyl)ethynyl]-2-methyl-3a,4,5,11b-tetrahydrochromeno[3,2-e]isoindole-1,3,11(2H)-trione (5c): M.p. 203-204 ${ }^{\circ} \mathrm{C}$. ${ }^{1} \mathrm{H}$ NMR (300 MHz, $\mathrm{CDCl}_{3}$ ): $\delta=2.97\left(\mathrm{~s}, 3 \mathrm{H}, \mathrm{NCH}_{3}\right.$ ), 3.02-3.07 (m, $2 \mathrm{H}, 5-\mathrm{H}), 3.28(\mathrm{dd}, J=7.6,5.0 \mathrm{~Hz}, 1 \mathrm{H}, 3 \mathrm{a}-\mathrm{H}), 3.71-3.75(\mathrm{~m}$, $1 \mathrm{H}, 4-\mathrm{H}), 3.75$ (s, $\left.3 \mathrm{H}, \mathrm{OCH}_{3}\right), 4.37$ (d, $\left.J=7.6 \mathrm{~Hz}, 1 \mathrm{H}, 11 \mathrm{~b}-\mathrm{H}\right)$, $6.73\left(\mathrm{~d}, J=8.9 \mathrm{~Hz}, 2 \mathrm{H}, 3^{\prime \prime}, 5^{\prime \prime}-\mathrm{H}\right), 7.20\left(\mathrm{~d}, J=8.9 \mathrm{~Hz}, 2 \mathrm{H}, 2^{\prime \prime}, 6^{\prime \prime}-\right.$ H), 7.39-7.44 (m, 2 H, 7-H, 9-H), 7.66 (ddd, $J=8.6,7.1,1.7 \mathrm{~Hz}$, $1 \mathrm{H}, 8-\mathrm{H}), 8.29(\mathrm{dd}, J=8.2,1.7 \mathrm{~Hz}, 1 \mathrm{H}, 10-\mathrm{H}) \mathrm{ppm} .{ }^{13} \mathrm{C} \mathrm{NMR}$ (75 MHz, $\left.\mathrm{CDCl}_{3}\right): \delta=24.7\left(\mathrm{NCH}_{3}\right), 27.8(\mathrm{C}-4), 33.3(\mathrm{C}-5), 37.7$ (C-11b), 43.3 (C-3a), $55.2\left(\mathrm{OCH}_{3}\right), 83.3\left(\mathrm{C}-1^{\prime}\right), 85.7\left(\mathrm{C}-2^{\prime}\right), 112.1$ (C-11a), 113.7 (C-1' ), 113.8 (C-3"' ,5'), 117.6 (C-7), 123.5 (C-10a), 125.2 (C-9), 126.4 (C-10), 133.0 (C-2' ,6 ') 133.7 (C-8), 155.7 (C6a), 159.7 (C-4' $), 162.3$ (C-5a), 174.3 (C-1), 176.2 (C-3 and C11) ppm. HRMS $\left(\mathrm{ESI}^{+}\right): m / z$ calcd. for $\mathrm{C}_{25} \mathrm{H}_{20} \mathrm{NO}_{5}[\mathrm{M}+\mathrm{H}]^{+}$ 414.1341, found 414.1329. MS $\left(\mathrm{ESI}^{+}\right): m / z(\%)=414(22)[\mathrm{M}+$ $\mathrm{H}]^{+}, 436(38)[\mathrm{M}+\mathrm{Na}]^{+}, 849(100)[2 \mathrm{M}+\mathrm{Na}]^{+}$.

4-[(4-Bromophenyl)ethynyl]-2-methyl-3a,4,5,11b-tetrahydrochromeno[3,2-e]isoindole-1,3,11(2H)-trione (5d): M.p. $214-215{ }^{\circ} \mathrm{C}$. ${ }^{1} \mathrm{H} \mathrm{NMR}\left(300 \mathrm{MHz}, \mathrm{CDCl}_{3}\right): \delta=2.96\left(\mathrm{~s}, 3 \mathrm{H}, \mathrm{CH}_{3}\right), 3.03-3.08(\mathrm{~m}$, $2 \mathrm{H}, 5-\mathrm{H}), 3.29(\mathrm{dd}, J=7.6,5.0 \mathrm{~Hz}, 1 \mathrm{H}, 3 \mathrm{a}-\mathrm{H}), 3.70-3.75(\mathrm{~m}, 1$ $\mathrm{H}, 4-\mathrm{H}), 4.39$ (d, $J=7.6 \mathrm{~Hz}, 1 \mathrm{H}, 11 \mathrm{~b}-\mathrm{H}), 7.14$ (d, $J=8.5 \mathrm{~Hz}, 2$ $\left.\mathrm{H}, 2^{\prime \prime}-\mathrm{H}, 6^{\prime \prime}-\mathrm{H}\right), 7.36$ (d, $\left.J=8.5 \mathrm{~Hz}, 2 \mathrm{H}, 3^{\prime \prime}-\mathrm{H}, 5^{\prime \prime}-\mathrm{H}\right), 7.40-7.45$ (m, 2 H, 7-H, 9-H), 7.67 (ddd, $J=8.5,7.1,1.6 \mathrm{~Hz}, 1 \mathrm{H}, 8-\mathrm{H}), 8.29$ (dd, $J=8.2,1.6 \mathrm{~Hz}, 1 \mathrm{H}, 10-\mathrm{H}) \mathrm{ppm} .{ }^{13} \mathrm{C} \mathrm{NMR}\left(75 \mathrm{MHz}, \mathrm{CDCl}_{3}\right)$ : $\delta=24.8\left(\mathrm{NCH}_{3}\right), 27.8(\mathrm{C}-4), 33.0(\mathrm{C}-5), 37.8(\mathrm{C}-11 \mathrm{~b}), 43.2(\mathrm{C}-3 \mathrm{a})$, $84.8\left(\mathrm{C}-1^{\prime}\right), 86.0\left(\mathrm{C}-2^{\prime}\right), 112.1$ (C-11a), 117.6 (C-7), $120.6\left(\mathrm{C}-1^{\prime \prime}\right)$, 123.0 (C-4"'), 123.8 (C-10a), 125.3 (C-9), 126.4 (C-10), 131.5 (C$3^{\prime \prime}$ and C-5"'), 133.0 (C-2" and C-6"'), 133.8 (C-8), 155.7 (C-6a), 162.3 (C-5a), 175.9 (C-1), 176.0 (C-3 and C-11) ppm. HRMS (ESI ${ }^{+}$): $\mathrm{m} / z$ calcd. for $\mathrm{C}_{24} \mathrm{H}_{17}{ }^{79} \mathrm{BrNO}_{4}[\mathrm{M}+\mathrm{H}]^{+} 462.0341$, found 462.0325; calcd. for $\mathrm{C}_{24} \mathrm{H}_{17}{ }^{81} \mathrm{BrNO}_{4}[\mathrm{M}+\mathrm{H}]^{+} 464.0320$, found 464.0302. $\mathrm{MS}\left(\mathrm{ESI}^{+}\right): \mathrm{m} / z(\%)=462(35)[\mathrm{M}+\mathrm{H}]^{+}, 947(100)[2 \mathrm{M}$ $+\mathrm{Na}+2 \mathrm{H}]^{+}$.

4-[(4-Methoxyphenyl)ethynyl]-2-phenyl-3a,4,5,11b-tetrahydrochromeno[3,2-e]isoindole-1,3,11(2H)-trione (5e): M.p. $164-166{ }^{\circ} \mathrm{C}$. ${ }^{1} \mathrm{H}$ NMR (300 MHz, $\mathrm{CDCl}_{3}$ ): $\delta=3.10-3.13(\mathrm{~m}, 2 \mathrm{H}, 5-\mathrm{H}), 3.37$ (dd, $J=7.7,4.9 \mathrm{~Hz}, 1 \mathrm{H}, 3-\mathrm{H}), 3.71\left(\mathrm{~s}, 3 \mathrm{H}, 4^{\prime \prime}-\mathrm{OCH}_{3}\right), 3.87-3.90$ (m, $1 \mathrm{H}, 4-\mathrm{H}), 4.56(\mathrm{~d}, J=4.9 \mathrm{~Hz}, 1 \mathrm{H}, 11 \mathrm{~b}-\mathrm{H}), 6.64(\mathrm{~d}, J=6.9 \mathrm{~Hz}$, $\left.2 \mathrm{H}, 3^{\prime \prime}, 5^{\prime \prime}-\mathrm{H}\right), 7.08\left(\mathrm{~d}, J=6.9 \mathrm{~Hz}, 2 \mathrm{H}, 2^{\prime \prime}, 6^{\prime \prime}-\mathrm{H}\right), 7.27-7.34$ (m, $\left.5 \mathrm{H}, 2^{\prime \prime \prime}, 3^{\prime \prime \prime}, 4^{\prime \prime \prime}, 5^{\prime \prime \prime}, 6^{\prime \prime \prime}-\mathrm{H}\right), 7.39-7.43(\mathrm{~m}, 1 \mathrm{H}, 9-\mathrm{H}), 7.43$ (d, $J$ $=7.7 \mathrm{~Hz}, 1 \mathrm{H}, 7-\mathrm{H}), 7.66(\mathrm{ddd}, J=7.8,7.7,1.6 \mathrm{~Hz}, 1 \mathrm{H}, 8-\mathrm{H})$, $8.28(\mathrm{dd}, J=8.3,1.6 \mathrm{~Hz}, 1 \mathrm{H}, 10-\mathrm{H}) \mathrm{ppm} .{ }^{13} \mathrm{C} \mathrm{NMR}(75 \mathrm{MHz}$, $\left.\mathrm{CDCl}_{3}\right): \delta=27.9(\mathrm{C}-4), 33.8(\mathrm{C}-5), 38.1(\mathrm{C}-11 \mathrm{~b}), 43.0$ (C-3a), 55.2 $\left(\mathrm{OCH}_{3}\right), 83.5\left(\mathrm{C}-1^{\prime}\right), 86.5\left(\mathrm{C}-2^{\prime}\right), 112.0(\mathrm{C}-11 \mathrm{a}), 113.66\left(\mathrm{C}-1^{\prime \prime}\right)$, 
113.73 (C-3"',5"'), 117.7 (C-7), 123.6 (C-10a), 125.3 (C-9), 126.4

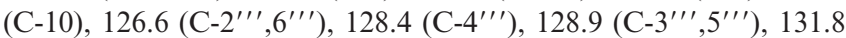
(C-1"' ), 133.3 (C-2"',6"), 133.7 (C-8), 155.8 (C-6a), 159.7 (C-4"'), 162.4 (C-5a), 173.0 (C-3), 175.3 (C-1), 176.2 (C-11) ppm.

General Procedure for the Synthesis of 4-(Arylethynyl)-2-methylchromeno[3,2-e]isoindole-1,3,11(2H)-triones 6a-d: 2,3-Dichloro-5,6dicyanobenzoquinone (DDQ) $(121 \mathrm{mg}, 534 \mu \mathrm{mol})$ was added to a solution of the appropriate 4-(arylethynyl)-2-methyl-3a,4,5,11btetrahydrochromeno[3,2-e]isoindole-1,3,11(2H)-triones 5a-d $(178 \mu \mathrm{mol})$ in toluene $(10 \mathrm{~mL})$. The mixture was stirred at $100{ }^{\circ} \mathrm{C}$ for $1 \mathrm{~h}$. After that period, the solvent was evaporated to dryness and the residue was purified by preparative TLC using $\mathrm{CH}_{2} \mathrm{Cl}_{2}$ as eluent to give desired 4-(arylethynyl)-2-methylchromeno[3,2-e] isoindole-1,3,11-triones $\mathbf{6 a - d}$ in good yields (57-83\%).

2-Methyl-4-(phenylethynyl)chromeno[3,2-e]isoindole-1,3,11(2H)trione (6a): Yield $42.5 \mathrm{mg}(63 \%)$, m.p. $273-274{ }^{\circ} \mathrm{C} .{ }^{1} \mathrm{H}$ NMR $\left(300 \mathrm{MHz}, \mathrm{CDCl}_{3}\right): \delta=3.26\left(\mathrm{~s}, 3 \mathrm{H}, \mathrm{CH}_{3}\right), 7.42-7.47$ (m, $4 \mathrm{H}, 9-$ $\left.\mathrm{H}, 3^{\prime \prime}, 4^{\prime \prime}, 5^{\prime \prime}-\mathrm{H}\right), 7.50$ (dd, $\left.J=8.3,0.6 \mathrm{~Hz}, 1 \mathrm{H}, 7-\mathrm{H}\right), 7.71-7.73$ (m, $\left.2 \mathrm{H}, 2^{\prime \prime}, 6^{\prime \prime}-\mathrm{H}\right), 7.77$ (ddd, $J=8.3,7.1,1.6 \mathrm{~Hz}, 1 \mathrm{H}, 8-\mathrm{H}$ ), 7.89 (s, $1 \mathrm{H}, 5-\mathrm{H}), 8.36(\mathrm{dd}, J=8.0,1.6 \mathrm{~Hz}, 1 \mathrm{H}, 10-\mathrm{H}) \mathrm{ppm} .{ }^{13} \mathrm{C} \mathrm{NMR}$ $\left(75 \mathrm{MHz}, \mathrm{CDCl}_{3}\right): \delta=24.5\left(\mathrm{NCH}_{3}\right), 84.0\left(\mathrm{C}-1^{\prime}\right), 100.4\left(\mathrm{C}-2^{\prime}\right), 117.6$ (C-7), 118.9 (C-11a), 121.8 (C-11b), 123.0 (C-10a), 124.8 (C-4), 125.1 (C-9), 127.0 (C-5), 127.4 (C-10), 128.6 (C-3" ,5" ), 128.8 (C-

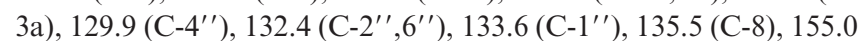
(C-6a), 159.6 (C-5a), 164.1 and 165.9 (C-1 and C-3), 174.0 (C11) ppm. HRMS (ESI): $\mathrm{m} / z$ calcd. for $\mathrm{C}_{24} \mathrm{H}_{14} \mathrm{NO}_{4}[\mathrm{M}+\mathrm{H}]^{+}$ 380.0923, found 380.0907. MS $\left(\mathrm{ESI}^{+}\right): \mathrm{m} / z(\%)=380(40)[\mathrm{M}+\mathrm{H}]$ ${ }^{+}, 402(21)[\mathrm{M}+\mathrm{Na}]^{+}, 781(100)[2 \mathrm{M}+\mathrm{Na}]^{+}, 797(27)[2 \mathrm{M}+$ $\mathrm{K}]^{+}$.

2-Methyl-4-[(4-methylphenyl)ethynyl]chromeno[3,2-e]isoindole1,3,11(2H)-trione (6b): Yield $53.9 \mathrm{mg}(77 \%)$, m.p. $326-327^{\circ} \mathrm{C} .{ }^{1} \mathrm{H}$ NMR (300 MHz, $\left.\mathrm{CDCl}_{3}\right): \delta=2.41\left(\mathrm{~s}, 3 \mathrm{H}, \mathrm{CH}_{3}\right), 3.26(\mathrm{~s}, 3 \mathrm{H}, \mathrm{N}-$ $\left.\mathrm{CH}_{3}\right), 7.24\left(\mathrm{~d}, J=8.0 \mathrm{~Hz}, 2 \mathrm{H}, 3^{\prime \prime}, 5^{\prime \prime}-\mathrm{H}\right), 7.45(\mathrm{t}, J=7.8 \mathrm{~Hz}, 1 \mathrm{H}$, 9-H), $7.50(\mathrm{~d}, J=8.3 \mathrm{~Hz}, 1 \mathrm{H}, 7-\mathrm{H}), 7.62(\mathrm{~d}, J=8.0 \mathrm{~Hz}, 2 \mathrm{H}$, $\left.2^{\prime \prime}, 6^{\prime \prime}-\mathrm{H}\right), 7.77$ (ddd, $\left.J=8.3,7.8,1.5 \mathrm{~Hz}, 1 \mathrm{H}, 8-\mathrm{H}\right), 7.88(\mathrm{~s}, 1 \mathrm{H}$, $5-\mathrm{H}), 8.37(\mathrm{dd}, J=7.8,1.5 \mathrm{~Hz}, 1 \mathrm{H}, 10-\mathrm{H}) \mathrm{ppm} .{ }^{13} \mathrm{C} \mathrm{NMR}$ $\left(500 \mathrm{MHz}, \mathrm{CDCl}_{3}\right): \delta=21.8\left(\mathrm{CH}_{3}\right), 24.5\left(\mathrm{NCH}_{3}\right), 83.7\left(\mathrm{C}-1^{\prime}\right)$, 100.9 (C-2'), 117.6 (C-7), 118.7 (C-1' $), 118.8$ (C-3a), 123.0 (C-10a), 125.07 (C-11b), 125.12 (C-9), 126.8 (C-5), 127.4 (C-10), 128.7 (C11a), $129.4\left(\mathrm{C}-3^{\prime \prime}, 5^{\prime \prime}\right), 132.3\left(\mathrm{C}-2^{\prime \prime}, 6^{\prime \prime}\right), 133.6$ (C-4), 135.4 (C-8), 140.5 (C-4' $), 155.0$ (C-6a), 159.7 (C-5a), 164.1 and 166.0 (C-1 and C-3), 174.1 (C-11) ppm. HRMS (ESI $\left.{ }^{+}\right): m / z$ calcd. for $\mathrm{C}_{25} \mathrm{H}_{16} \mathrm{NO}_{4}$ $[\mathrm{M}+\mathrm{H}]^{+}$394.1079, found 394.1060. MS $\left(\mathrm{ESI}^{+}\right): \mathrm{m} / \mathrm{z}(\%)=394$ (40) $[\mathrm{M}+\mathrm{H}]^{+}, 416(72)[\mathrm{M}+\mathrm{Na}]^{+}, 432(33)[\mathrm{M}+\mathrm{K}]^{+}, 809(100)$ $[2 \mathrm{M}+\mathrm{Na}]^{+}, 825(43)[2 \mathrm{M}+\mathrm{K}]^{+}$.

4-[(4-Methoxyphenyl)ethynyl]-2-methylchromeno[3,2-e]isoindole1,3,11(2H)-trione (6c): Yield $60.5 \mathrm{mg}(83 \%)$, m.p. $295-297{ }^{\circ} \mathrm{C} .{ }^{1} \mathrm{H}$ NMR (500 MHz, $\left.\mathrm{CDCl}_{3}\right): \delta=3.26\left(\mathrm{~s}, 3 \mathrm{H}, \mathrm{NCH}_{3}\right), 3.87(\mathrm{~s}, 3 \mathrm{H}$, $\left.\mathrm{OCH}_{3}\right), 6.95\left(\mathrm{~d}, J=8.9 \mathrm{~Hz}, 2 \mathrm{H}, 3^{\prime \prime}, 5^{\prime \prime}-\mathrm{H}\right), 7.44(\mathrm{ddd}, J=7.8,7.3$, $1.0 \mathrm{~Hz}, 1 \mathrm{H}, 9-\mathrm{H}), 7.50(\mathrm{dd}, J=8.4,1.0 \mathrm{~Hz}, 1 \mathrm{H}, 7-\mathrm{H}), 7.68(\mathrm{~d}, J$ $\left.=8.9 \mathrm{~Hz}, 2 \mathrm{H}, 2^{\prime \prime}, 6^{\prime \prime}-\mathrm{H}\right), 7.77$ (ddd, $J=8.4,7.3,1.6 \mathrm{~Hz}, 1 \mathrm{H}, 8-$ H), 7.85 (s, $1 \mathrm{H}, 5-\mathrm{H}), 8.37$ (dd, $J=7.8,1.6 \mathrm{~Hz}, 1 \mathrm{H}, 10-\mathrm{H}) \mathrm{ppm}$. ${ }^{13} \mathrm{C} \mathrm{NMR}\left(126 \mathrm{MHz}, \mathrm{CDCl}_{3}\right): \delta=24.5\left(\mathrm{NCH}_{3}\right), 55.4\left(\mathrm{OCH}_{3}\right), 83.4$ $\left(\mathrm{C}-1^{\prime}\right), 101.2\left(\mathrm{C}-2^{\prime}\right), 113.8\left(\mathrm{C}-1^{\prime \prime}\right), 114.3\left(\mathrm{C}-3^{\prime \prime}, 5^{\prime \prime}\right), 117.6(\mathrm{C}-7)$, 118.6 (C-11a), 123.0 (C-10a), 125.1 (C-9), 125.3 (C-11b), 126.5 (C5), 127.4 (C-10), 128.5 (C-3a), 133.6 (C-4), $134.2\left(\mathrm{C}-2^{\prime \prime}, 6^{\prime \prime}\right), 135.4$ (C-8), 155.0 (C-6a), 159.7 (C-5a), 161.0 (C-4"'), 164.2 and 166.1 (C-1 and C-3), 174.1 (C-11) ppm. HRMS (ESI $\left.{ }^{+}\right): \mathrm{m} / \mathrm{z}$ calcd. for $\mathrm{C}_{25} \mathrm{H}_{16} \mathrm{NO}_{5}[\mathrm{M}+\mathrm{H}]^{+}$410.1028, found 410.1007. MS (ESI $\left.{ }^{+}\right): \mathrm{m} / \mathrm{z}$ $(\%)=410(14)[\mathrm{M}+\mathrm{H}]^{+}, 432(30)[\mathrm{M}+\mathrm{Na}]^{+}, 448(15)[\mathrm{M}+\mathrm{K}]^{+}$, $841(100)[2 \mathrm{M}+\mathrm{Na}]^{+}, 857(47)[2 \mathrm{M}+\mathrm{K}]^{+}$.
4-[(4-Bromophenyl)ethynyl]-2-methylchromeno[3,2-e]isoindole1,3,11(2H)-trione (6d): Yield $46.5 \mathrm{mg}(57 \%)$, m.p. $332-333{ }^{\circ} \mathrm{C} .{ }^{1} \mathrm{H}$ NMR (500 MHz, $\left.\mathrm{CDCl}_{3}\right): \delta=3.26\left(\mathrm{~s}, 3 \mathrm{H}, \mathrm{NCH}_{3}\right), 7.45$ (ddd, $J=$ $8.0,7.1,0.9 \mathrm{~Hz}, 1 \mathrm{H}, 9-\mathrm{H}), 7.51(\mathrm{~d}, J=8.2 \mathrm{~Hz}, 1 \mathrm{H}, 7-\mathrm{H}), 7.56$ 7.60 (m, $\left.4 \mathrm{H}, 2^{\prime \prime}, 6^{\prime \prime}-\mathrm{H}, 3^{\prime \prime}, 5^{\prime \prime}-\mathrm{H}\right), 7.78$ (ddd, $J=8.2,7.1,1.6 \mathrm{~Hz}$, $1 \mathrm{H}, 8-\mathrm{H}), 7.88$ (s, $1 \mathrm{H}, 5-\mathrm{H}), 8.37$ (dd, $J=8.0,1.6 \mathrm{~Hz}, 1 \mathrm{H}, 10-$ $\mathrm{H})$ ppm. ${ }^{13} \mathrm{C} \mathrm{NMR}\left(126 \mathrm{MHz}, \mathrm{CDCl}_{3}\right): \delta=24.5\left(\mathrm{NCH}_{3}\right), 85.0(\mathrm{C}-$ $\left.1^{\prime}\right), 99.0$ (C-2'), 117.6 (C-7), 119.1 (C-11a), 120.7 (C-1'”), 123.0 (C10a), 124.3 and 124.5 (C-4 and C-11b), 125.2 (C-9), 126.9 (C-5), 127.5 (C-10), 128.8 (C-3a), $132.0\left(\mathrm{C}-3^{\prime \prime}, 5^{\prime \prime}\right), 133.5$ (C-4' $), 133.7$ (C-2" $\left.{ }^{\prime \prime} 6^{\prime \prime}\right), 135.5$ (C-8), 155.0 (C-6a), 159.7 (C-5a), 164.1 and 166.2 (C-1 and C-3), $174.0(\mathrm{C}-11)$ ppm. HRMS $\left(\mathrm{ESI}^{+}\right): \mathrm{m} / \mathrm{z}$ calcd. for $\mathrm{C}_{24} \mathrm{H}_{13}{ }^{79} \mathrm{BrNO}_{4}[\mathrm{M}+\mathrm{H}]^{+}$458.0028, found 458.0006; calcd. for $\mathrm{C}_{24} \mathrm{H}_{13}{ }^{81} \mathrm{BrNO}_{4}[\mathrm{M}+\mathrm{H}]^{+}$460.0007, found 459.9986. MS $\left(\mathrm{ESI}^{+}\right)$: $m / z(\%)=480(23)[\mathrm{M}+\mathrm{Na}]^{+}, 497(12)[\mathrm{M}+\mathrm{K}]^{+}, 939(19)[2 \mathrm{M}+$ $\mathrm{Na}]^{+}$.

General Procedure for the Synthesis of 4-(4-Aryl-2H-1,2,3-triazol-5yl)-2-methylchromeno[3,2-e]isoindole-1,3,11(2H)-triones 7a-d: Sodium azide ( $43 \mathrm{mg}, 660 \mu \mathrm{mol})$ was added to a solution of the appropriate 4-(arylethynyl)-2-methylchromeno[3,2-e]isoindole$1,3,11(2 H)$-trione $\mathbf{6 a}-\mathbf{d}(132 \mu \mathrm{mol})$ in dry DMF $(5 \mathrm{~mL})$. The mixture was stirred under reflux for $1 \mathrm{~h}$ under nitrogen atmosphere. After that period, the solution was poured into ice $(30 \mathrm{~g})$ and water $(30 \mathrm{~mL})$, and the $\mathrm{pH}$ was adjusted to 4 with diluted $\mathrm{HCl}$. The mixture was vigorously stirred for $15 \mathrm{~min}$, the precipitate was removed by filtration, washed with water $(3 \times 20 \mathrm{~mL})$ and light petroleum $(3 \times 20 \mathrm{~mL})$, and recrystallized from ethanol to afford desired xanthone-1,2,3-triazole dyads $7 \mathbf{a}-\mathbf{d}$ in excellent yields (88-90\%).

2-Methyl-4-(4-phenyl-2H-1,2,3-triazol-5-yl)chromeno[3,2-e]isoindole-1,3,11(2H)-trione (7a): Yield $49.6 \mathrm{mg}(89 \%)$, m.p. 304 $306{ }^{\circ} \mathrm{C} .{ }^{1} \mathrm{H}$ NMR $\left[500 \mathrm{MHz},\left(\mathrm{CD}_{3}\right)_{2} \mathrm{SO}+\mathrm{TFA}\right]: \delta=2.94(\mathrm{~s}, 3 \mathrm{H}$, $\left.\mathrm{NCH}_{3}\right), 7.30-7.34\left(\mathrm{~m}, 3 \mathrm{H}, 3^{\prime \prime}, 4^{\prime \prime}, 5^{\prime \prime}-\mathrm{H}\right), 7.47$ (dd, $J=7.8,1.7 \mathrm{~Hz}$, $\left.2 \mathrm{H}, 2^{\prime \prime}, 6^{\prime \prime}-\mathrm{H}\right), 7.55$ (ddd, $\left.J=7.7,7.3,0.9 \mathrm{~Hz}, 1 \mathrm{H}, 9-\mathrm{H}\right), 7.68$ (d, $J=8.4 \mathrm{~Hz}, 1 \mathrm{H}, 7-\mathrm{H}), 7.91$ (ddd, $J=8.4,7.3,1.5 \mathrm{~Hz}, 1 \mathrm{H}, 8-\mathrm{H})$, 8.04 (s, $1 \mathrm{H}, 5-\mathrm{H}), 8.24$ (dd, $J=7.7,1.5 \mathrm{~Hz}, 1 \mathrm{H}, 10-\mathrm{H}) \mathrm{ppm} .{ }^{13} \mathrm{C}$ NMR [126 MHz, $\left.\left(\mathrm{CD}_{3}\right)_{2} \mathrm{SO}+\mathrm{TFA}\right]: \delta=24.2\left(\mathrm{NCH}_{3}\right), 118.0(\mathrm{C}-$ 7), 119.4 (C-11a), 122.7 (C-10a), 125.3 (C-9), 126.4 (C-5), 126.5 (C-

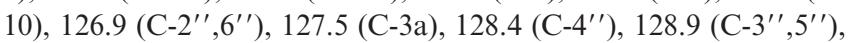
129.9 (C-1" $\left.{ }^{\prime \prime}\right), 133.7$ and 133.8 (C-4 and C-11b), 136.0 (C-8), 136.8 $\left(\mathrm{C}-5^{\prime}\right), 142.7$ (C-4'), 154.8 (C-6a), 159.5 (C-5a), 163.9 and 165.4 (C-1 and C-3), 173.6 (C-11) ppm. HRMS (ESI $\left.{ }^{+}\right): m / z$ calcd. for $\mathrm{C}_{24} \mathrm{H}_{15} \mathrm{~N}_{4} \mathrm{O}_{4}[\mathrm{M}+\mathrm{H}]^{+}$423.1093, found 423.1076. MS $\left(\mathrm{ESI}^{+}\right): \mathrm{m} / \mathrm{z}$ $(\%)=423(100)[\mathrm{M}+\mathrm{H}]^{+}, 445(10)[\mathrm{M}+\mathrm{Na}]^{+}$.

2-Methyl-4-[4-(4-methylphenyl)-2H-1,2,3-triazol-5-yl]chromeno[3,2-e isoindole-1,3,11(2H)-trione (7b): Yield $51.8 \mathrm{mg}$ (90\%), m.p. 318-320 ${ }^{\circ} \mathrm{C} .{ }^{1} \mathrm{H}$ NMR [500 MHz, $\left(\mathrm{CD}_{3}\right)_{2} \mathrm{SO}+\mathrm{TFA}$ ]: $\delta=2.23(\mathrm{~s}, 3$ $\left.\mathrm{H}, \mathrm{CH}_{3}\right), 2.92$ (s, $\left.3 \mathrm{H}, \mathrm{NCH}_{3}\right), 7.09$ (d, $\left.J=8.1 \mathrm{~Hz}, 2 \mathrm{H}, 3^{\prime \prime}, 5^{\prime \prime}-\mathrm{H}\right)$, $7.32\left(\mathrm{~d}, J=8.1 \mathrm{~Hz}, 2 \mathrm{H}, 2^{\prime \prime}, 6^{\prime \prime}-\mathrm{H}\right), 7.49$ (t, $\left.J=7.6 \mathrm{~Hz}, 1 \mathrm{H}, 9-\mathrm{H}\right)$, $7.61(\mathrm{~d}, J=8.0 \mathrm{~Hz}, 1 \mathrm{H}, 7-\mathrm{H}), 7.86(\mathrm{ddd}, J=8.0,7.6,1.6 \mathrm{~Hz}, 1$ H, 8-H), $7.96(\mathrm{~s}, 1 \mathrm{H}, 5-\mathrm{H}), 8.21(\mathrm{dd}, J=7.6,1.6 \mathrm{~Hz}, 1 \mathrm{H}, 10-$ $\mathrm{H}) \mathrm{ppm} .{ }^{13} \mathrm{C} \mathrm{NMR}\left[126 \mathrm{MHz},\left(\mathrm{CD}_{3}\right)_{2} \mathrm{SO}+\mathrm{TFA}\right]: \delta=20.9\left(\mathrm{CH}_{3}\right)$, $24.3\left(\mathrm{NCH}_{3}\right), 118.2(\mathrm{C}-7), 119.7$ (C-11a), 123.0 (C-10a), $125.5(\mathrm{C}-$ 9), 126.6 (C-5), 126.8 (C-10), $127.1\left(\mathrm{C}-2^{\prime \prime}, 6^{\prime \prime}\right), 127.2\left(\mathrm{C}-1^{\prime \prime}\right), 127.8$

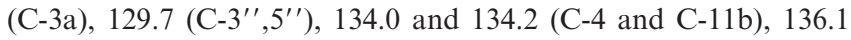
(C-8), 136.8 (C-5'), 138.2 (C-4' '), 142.6 (C-4'), 155.1 (C-6a), 159.8 (C-5a), 164.2 and 165.7 (C-1 and C-3), 173.9 (C-11) ppm. HRMS $\left(\mathrm{ESI}^{+}\right): \mathrm{m} / z$ calcd. for $\mathrm{C}_{25} \mathrm{H}_{17} \mathrm{~N}_{4} \mathrm{O}_{4}[\mathrm{M}+\mathrm{H}]^{+} 437.1250$, found 437.1228. $\mathrm{MS}\left(\mathrm{ESI}^{+}\right): m / z(\%)=437(100)[\mathrm{M}+\mathrm{H}]^{+}, 459(71)[\mathrm{M}$ $+\mathrm{Na}]^{+}, 475(38)[\mathrm{M}+\mathrm{K}]^{+}, 895(78)[2 \mathrm{M}+\mathrm{Na}]^{+}$

4-[4-(4-Methoxyphenyl)-2H-1,2,3-triazol-5-yl]-2-methylchromeno[3,2-e]isoindole-1,3,11(2H)-trione (7c): Yield $52.5 \mathrm{mg}$ (88\%), m.p. 297-299 ${ }^{\circ} \mathrm{C} .{ }^{1} \mathrm{H}$ NMR [500 MHz, $\left.\left(\mathrm{CD}_{3}\right)_{2} \mathrm{SO}+\mathrm{TFA}\right]: \delta=2.93(\mathrm{~s}, 3$ 
$\left.\mathrm{H}, \mathrm{NCH}_{3}\right), 3.69\left(\mathrm{~s}, 3 \mathrm{H}, \mathrm{OCH}_{3}\right), 6.84\left(\mathrm{~d}, J=8.9 \mathrm{~Hz}, 2 \mathrm{H}, 3^{\prime \prime}, 5^{\prime \prime}-\right.$ $\mathrm{H}), 7.36\left(\mathrm{~d}, J=8.9 \mathrm{~Hz}, 2 \mathrm{H}, 2^{\prime \prime}, 6^{\prime \prime}-\mathrm{H}\right), 7.50$ (ddd, $J=7.8,7.3$, $0.8 \mathrm{~Hz}, 1 \mathrm{H}, 9-\mathrm{H}), 7.63$ (d, $J=8.3 \mathrm{~Hz}, 1 \mathrm{H}, 7-\mathrm{H}), 7.87$ (ddd, $J=$ 8.3, 7.3, $1.5 \mathrm{~Hz}, 1 \mathrm{H}, 8-\mathrm{H}), 7.97$ (s, $1 \mathrm{H}, 5-\mathrm{H}), 8.22$ (dd, $J=7.8$, $1.5 \mathrm{~Hz}, 1 \mathrm{H}, 10-\mathrm{H}) \mathrm{ppm} .{ }^{13} \mathrm{C} \mathrm{NMR}\left[126 \mathrm{MHz},\left(\mathrm{CD}_{3}\right)_{2} \mathrm{SO}+\mathrm{TFA}\right]$ : $\delta=24.2\left(\mathrm{NCH}_{3}\right), 55.2\left(\mathrm{OCH}_{3}\right), 114.5\left(\mathrm{C}-3^{\prime \prime}, 5^{\prime \prime}\right), 118.1(\mathrm{C}-7), 119.5$ (C-11a), 122.2 (C-1' $), 122.9$ (C-10a), 125.4 (C-9), 126.5 (C-5), 126.6 (C-10), 127.7 (C-3a), $128.4\left(\mathrm{C}-2^{\prime \prime}, 6^{\prime \prime}\right), 133.8$ and 134.2 (C-4 and C-11b), 136.1 (C-8), 136.3 (C-5'), 142.1 (C-4'), 154.9 (C-6a), 159.6 and 159.7 (C-4" and C-5a), 164.1 and 165.6 (C-1 and C-3), 173.8 (C-11) ppm. HRMS (ESI $\left.{ }^{+}\right): m / z$ calcd. for $\mathrm{C}_{25} \mathrm{H}_{17} \mathrm{~N}_{4} \mathrm{O}_{5}[\mathrm{M}$ $+\mathrm{H}]^{+}$453.1199, found 453.1177. MS $\left(\mathrm{ESI}^{+}\right): \mathrm{m} / z(\%)=437(16)$ $\left[\mathrm{M}-\mathrm{CH}_{3}\right]^{+}, 453(100)[\mathrm{M}+\mathrm{H}]^{+}, 475(77)[\mathrm{M}+\mathrm{Na}]^{+}, 491(33)[\mathrm{M}$ $+\mathrm{K}]^{+}, 927(64)[2 \mathrm{M}+\mathrm{Na}]^{+}$.

4-[4-(4-Bromophenyl)-2H-1,2,3-triazol-5-yl]-2-methylchromeno[3,2-e]isoindole-1,3,11(2H)-trione (7d): Yield $59.5 \mathrm{mg}$ (90\%), m.p. 319-320 ${ }^{\circ} \mathrm{C} .{ }^{1} \mathrm{H}$ NMR [500 MHz, $\left.\left(\mathrm{CD}_{3}\right)_{2} \mathrm{SO}+\mathrm{TFA}\right]: \delta=2.92(\mathrm{~s}, 3$ $\left.\mathrm{H}, \mathrm{NCH}_{3}\right), 7.39\left(\mathrm{~d}, J=8.7 \mathrm{~Hz}, 2 \mathrm{H}, 2^{\prime \prime}, 6^{\prime \prime}-\mathrm{H}\right), 7.47(\mathrm{~d}, J=8.7 \mathrm{~Hz}$, $\left.2 \mathrm{H}, 3^{\prime \prime}, 5^{\prime \prime}-\mathrm{H}\right), 7.51$ (ddd, $\left.J=7.8,7.3,0.9 \mathrm{~Hz}, 1 \mathrm{H}, 9-\mathrm{H}\right), 7.64$ (dd, $J=8.2,0.9 \mathrm{~Hz}, 1 \mathrm{H}, 7-\mathrm{H}), 7.88$ (ddd, $J=8.2,7.3,1.6 \mathrm{~Hz}, 1 \mathrm{H}, 8-$ H), 8.02 (s, $1 \mathrm{H}, 5-\mathrm{H}), 8.22$ (dd, $J=7.8,1.6 \mathrm{~Hz}, 1 \mathrm{H}, 10-\mathrm{H}) \mathrm{ppm}$. ${ }^{13} \mathrm{C}$ NMR $\left[126 \mathrm{MHz},\left(\mathrm{CD}_{3}\right)_{2} \mathrm{SO}+\mathrm{TFA}\right]: \delta=24.3\left(\mathrm{NCH}_{3}\right), 118.2$ (C-7), 119.7 (C-11a), 121.9 (C-4" ), 122.9 (C-10a), 125.5 (C-9), 126.6 (C-5), 126.7 (C-10), 127.6 (C-3a), $129.0\left(\mathrm{C}-2^{\prime \prime}, 6^{\prime \prime}\right), 129.7$ (C$\left.1^{\prime \prime}\right), 132.1\left(\mathrm{C}-3^{\prime \prime}, 5^{\prime \prime}\right), 133.5$ and 134.0 (C-4 and C-11b), $136.2(\mathrm{C}-$ 8), $139.3\left(\mathrm{C}^{-} 5^{\prime}\right), 142.5\left(\mathrm{C}-4^{\prime}\right), 155.0$ (C-6a), 159.8 (C-5a), 164.1 and 165.7 (C-1 and C-3), 173.8 (C-11) ppm. HRMS (ESI $\left.{ }^{+}\right): \mathrm{m} / z$ calcd. for $\mathrm{C}_{24} \mathrm{H}_{14}{ }^{79} \mathrm{BrN}_{4} \mathrm{O}_{4}[\mathrm{M}+\mathrm{H}]^{+}$501.0198, found 501.0180; calcd. for $\mathrm{C}_{24} \mathrm{H}_{14}{ }^{81} \mathrm{BrN}_{4} \mathrm{O}_{4}[\mathrm{M}+\mathrm{H}]^{+}$503.0178, found 503.0155. MS $\left(\mathrm{ESI}^{+}\right)$: $m / z(\%)=501(100)[\mathrm{M}+\mathrm{H}]^{+}$.

General Procedure for the Synthesis of (E)-2-[2-(4-Aryl-2H-1,2,3triazol-5-yl)vinyl]-4H-chromen-4-ones $(\mathbf{8 a}-\mathbf{d})$ : Sodium azide $(117 \mathrm{mg}, 1.8 \mathrm{mmol})$ was added to a solution of the appropriate $(E)$ 2-(4-arylbut-1-en-3-yn-1-yl)-4H-chromen-4-ones 4a-d (367 $\mu$ mol) in dry DMF $(5 \mathrm{~mL})$. The mixture was stirred under reflux for $1 \mathrm{~h}$ under nitrogen atmosphere. After that period, the solution was poured into ice $(30 \mathrm{~g})$ and water $(30 \mathrm{~mL})$, and the $\mathrm{pH}$ was adjusted to 4 with diluted $\mathrm{HCl}$. The mixture was vigorously stirred for $15 \mathrm{~min}$, the precipitate was washed with water $(3 \times 20 \mathrm{~mL})$ and light petroleum $(3 \times 20 \mathrm{~mL})$, removed by filtration and recrystallized from ethanol to give (E)-2-[2-(4-aryl-2 $H$-1,2,3-triazol-5-yl) vinyl]-4H-chromen-4-ones 8a-d in excellent yields (90-97\%).

(E)-2-[2-(4-Phenyl-2 $H$-1,2,3-triazol-5-yl)vinyl]-4 $H$-chromen-4-one (8a): Yield $0.10 \mathrm{~g}(90 \%)$, m.p. $240-241{ }^{\circ} \mathrm{C} .{ }^{1} \mathrm{H}$ NMR $[300 \mathrm{MHz}$, $\left.\left(\mathrm{CD}_{3}\right)_{2} \mathrm{SO}+\mathrm{TFA}\right]: \delta=6.58(\mathrm{~s}, 1 \mathrm{H}, 3-\mathrm{H}), 7.35(\mathrm{~d}, J=15.8 \mathrm{~Hz}, 1$ $\mathrm{H}, \alpha-\mathrm{H}), 7.46(\mathrm{t}, J=7.7 \mathrm{~Hz}, 1 \mathrm{H}, 6-\mathrm{H}), 7.53(\mathrm{t}, J=7.5 \mathrm{~Hz}, 1 \mathrm{H}$, $\left.4^{\prime \prime}-\mathrm{H}\right), 7.61\left(\mathrm{t}, J=7.5 \mathrm{~Hz}, 2 \mathrm{H}, 3^{\prime \prime}, 5^{\prime \prime}-\mathrm{H}\right), 7.65(\mathrm{~d}, J=8.1 \mathrm{~Hz}, 1$ $\mathrm{H}, 8-\mathrm{H}), 7.68(\mathrm{~d}, J=15.8 \mathrm{~Hz}, 1 \mathrm{H}, \beta-\mathrm{H}), 7.74(\mathrm{~d}, J=7.5 \mathrm{~Hz}, 2 \mathrm{H}$, $\left.2^{\prime \prime}, 6^{\prime \prime}-\mathrm{H}\right), 7.77$ (ddd, $\left.J=8.1,7.7,1.5 \mathrm{~Hz}, 1 \mathrm{H}, 7-\mathrm{H}\right), 8.06(\mathrm{dd}, J=$ 7.7, $1.5 \mathrm{~Hz}, 1 \mathrm{H}, 5-\mathrm{H}) \mathrm{ppm} .{ }^{13} \mathrm{C} \mathrm{NMR}\left[126 \mathrm{MHz},\left(\mathrm{CD}_{3}\right)_{2} \mathrm{SO}+\right.$ TFA]: $\delta=111.1(\mathrm{C}-3), 118.7(\mathrm{C}-8), 123.1(\mathrm{C}-\alpha), 123.9(\mathrm{C}-4 \mathrm{a}), 124.0$ (C- $\beta$ ), 125.3 (C-5), 125.6 (C-6), $128.5\left(\mathrm{C}-2^{\prime \prime}, 6^{\prime \prime}\right), 129.3\left(\mathrm{C}-1^{\prime \prime}\right)$, 129.4 (C-4' $), 129.6$ (C-3' $\left.{ }^{\prime \prime} 5^{\prime \prime}\right), 134.5$ (C-7), 138.3 (C-4'), 142.6 (C5'), 156.0 (C-8a), 161.5 (C-2), 177.7 (C-4) ppm. HRMS (ESI $\left.{ }^{+}\right): \mathrm{m} / \mathrm{z}$ calcd. for $\mathrm{C}_{19} \mathrm{H}_{14} \mathrm{~N}_{3} \mathrm{O}_{2}[\mathrm{M}+\mathrm{H}]^{+} 316.1086$, found 316.1075. MS $\left(\mathrm{ESI}^{+}\right): m / z(\%)=(100) 316[\mathrm{M}+\mathrm{H}]^{+}, 653(22)[2 \mathrm{M}+\mathrm{Na}]^{+}$.

(E)-2-\{2-[4-(4-Methylphenyl)-2H-1,2,3-triazol-5-yl]vinyl $\}-4 H-$ chromen-4-one (8b): Yield $0.12 \mathrm{~g}(98 \%)$, m.p. $239-240{ }^{\circ} \mathrm{C} .{ }^{1} \mathrm{H}$ NMR [300 MHz, $\left.\left(\mathrm{CD}_{3}\right)_{2} \mathrm{SO}+\mathrm{TFA}\right]: \delta=2.42\left(\mathrm{~s}, 3 \mathrm{H}, \mathrm{CH}_{3}\right), 6.61(\mathrm{~s}, 1 \mathrm{H}$, 3-H), $7.34(\mathrm{~d}, J=15.8 \mathrm{~Hz}, 1 \mathrm{H}, \alpha-\mathrm{H}), 7.41(\mathrm{~d}, J=7.7 \mathrm{~Hz}, 2 \mathrm{H}$, $\left.3^{\prime \prime}, 5^{\prime \prime}-\mathrm{H}\right), 7.48$ (t, $\left.J=7.7 \mathrm{~Hz}, 1 \mathrm{H}, 6-\mathrm{H}\right), 7.61(\mathrm{~d}, J=7.7 \mathrm{~Hz}, 2 \mathrm{H}$, $\left.2^{\prime \prime}, 6^{\prime \prime}-\mathrm{H}\right), 7.64(\mathrm{~d}, J=15.8 \mathrm{~Hz}, 1 \mathrm{H}, \beta-\mathrm{H}), 7.70(\mathrm{~d}, J=8.0 \mathrm{~Hz}, 1$
H, 8-H), 7.80 (ddd, $J=8.0,7.7,1.4 \mathrm{~Hz}, 1 \mathrm{H}, 7-\mathrm{H}), 8.03$ (dd, $J=$ 7.7, $1.4 \mathrm{~Hz}, 1 \mathrm{H}, 5-\mathrm{H}) \mathrm{ppm} .{ }^{13} \mathrm{C}$ NMR $\left[126 \mathrm{MHz},\left(\mathrm{CD}_{3}\right)_{2} \mathrm{SO}+\right.$ TFA]: $\delta=21.0\left(\mathrm{CH}_{3}\right), 110.7(\mathrm{C}-3), 118.5(\mathrm{C}-8), 122.4(\mathrm{C}-\alpha), 123.6$ (C- $\beta$ ), 123.8 (C-4a), 124.9 (C-5), 125.4 (C-6), 125.7 (C-1" ), 128.1 (C-2" and C-6"'), 129.9 (C-3" and C-5"), 134.3 (C-7), 137.8 (C4'), 138.8 (C-4' ), 141.8 (C-5'), 155.5 (C-8a), 161.1 (C-2), 177.3 (C4) ppm. HRMS-EI: $m / z$ calcd. for $\mathrm{C}_{20} \mathrm{H}_{15} \mathrm{O}_{2} \mathrm{~N}_{3} \mathrm{M}^{+\cdot} 329.1164$, found 329.1166. MS $\left(\mathrm{ESI}^{+}\right): m / z(\%)=330(100)[\mathrm{M}+\mathrm{H}]^{+}, 352$ (15) $[\mathrm{M}+\mathrm{Na}]^{+}$.

(E)-2-\{2-[4-(4-Methoxyphenyl)-2H-1,2,3-triazol-5-yl]vinyl $\}-4 H$ chromen-4-one (8c): Yield 0.12 g (98\%), m.p. $237-238^{\circ} \mathrm{C} .{ }^{1} \mathrm{H}$ NMR [500 MHz, $\left.\left(\mathrm{CD}_{3}\right)_{2} \mathrm{SO}+\mathrm{TFA}\right]: \delta=3.85\left(\mathrm{~s}, 3 \mathrm{H}, \mathrm{OCH}_{3}\right), 6.60(\mathrm{~s}, 1$ $\mathrm{H}, 3-\mathrm{H}), 7.16$ (d, $\left.J=8.8 \mathrm{~Hz}, 2 \mathrm{H}, 3^{\prime \prime}, 5^{\prime \prime}-\mathrm{H}\right), 7.32$ (d, $J=15.8 \mathrm{~Hz}$, $1 \mathrm{H}, \alpha-\mathrm{H}), 7.46(\mathrm{ddd}, J=7.7,7.2,1.0 \mathrm{~Hz}, 1 \mathrm{H}, 6-\mathrm{H}), 7.62(\mathrm{~d}, J=$ $15.8 \mathrm{~Hz}, 1 \mathrm{H}, \beta-\mathrm{H}), 7.64\left(\mathrm{~d}, J=8.8 \mathrm{~Hz}, 2 \mathrm{H}, 2^{\prime \prime}, 6^{\prime \prime}-\mathrm{H}\right), 7.70(\mathrm{~d}, J$ $=8.1 \mathrm{~Hz}, 1 \mathrm{H}, 8-\mathrm{H}), 7.79(\mathrm{ddd}, J=8.1,7.2,1.6 \mathrm{~Hz}, 1 \mathrm{H}, 7-\mathrm{H})$, $8.01(\mathrm{dd}, J=7.7,1.6 \mathrm{~Hz}, 1 \mathrm{H}, 5-\mathrm{H}) \mathrm{ppm} .{ }^{13} \mathrm{C} \mathrm{NMR}[126 \mathrm{MHz}$, $\left.\left(\mathrm{CD}_{3}\right)_{2} \mathrm{SO}+\mathrm{TFA}\right]: \delta=55.4\left(\mathrm{OCH}_{3}\right), 110.6(\mathrm{C}-3), 114.8\left(\mathrm{C}-3^{\prime \prime}, 5^{\prime \prime}\right)$, 118.5 (C-8), 120.7 (C-1' $), 122.2$ (C- $\alpha), 123.6$ (C-4a), 123.9 (C- $\beta$ ), 124.9 (C-5), 125.4 (C-6), $129.6\left(\mathrm{C}-2^{\prime \prime}, 6^{\prime \prime}\right), 134.3$ (C-7), 137.6 (C$\left.4^{\prime}\right), 145.1$ (C-5'), 155.5 (C-8a), 160.1 (C-4'), 161.2 (C-2), 177.2 (C4) ppm. HRMS-EI: $\mathrm{m} / z$ calcd. for $\mathrm{C}_{20} \mathrm{H}_{15} \mathrm{O}_{3} \mathrm{~N}_{3} \mathrm{M}^{+\cdot} 345.1113$, found 345.1116. $\mathrm{MS}\left(\mathrm{ESI}^{+}\right): \mathrm{m} / z(\%)=346(100)[\mathrm{M}+\mathrm{H}]^{+}, 368$ (15) $[\mathrm{M}+\mathrm{Na}]^{+}$

(E)-2-\{2-[4-(4-Bromophenyl)-2 H-1,2,3-triazol-5-yl]vinyl\}-4Hchromen-4-one (8d): Yield $0.14 \mathrm{~g}(97 \%)$, m.p. $293-294{ }^{\circ} \mathrm{C} .{ }^{1} \mathrm{H}$ NMR [300 MHz, $\left.\left(\mathrm{CD}_{3}\right)_{2} \mathrm{SO}+\mathrm{TFA}\right]: \delta=6.58(\mathrm{~s}, 1 \mathrm{H}, 3-\mathrm{H}), 7.30(\mathrm{~d}, J=$ $15.8 \mathrm{~Hz}, 1 \mathrm{H}, \alpha-\mathrm{H}), 7.42$ (ddd, $J=7.7,7.4,1.3 \mathrm{~Hz}, 1 \mathrm{H}, 6-\mathrm{H}), 7.61$ $(\mathrm{d}, J=15.8 \mathrm{~Hz}, 1 \mathrm{H}, \beta-\mathrm{H}), 7.64\left(\mathrm{~d}, J=8.5 \mathrm{~Hz}, 2 \mathrm{H}, 2^{\prime \prime}, 6^{\prime \prime}-\mathrm{H}\right)$, $7.68(\mathrm{~d}, J=7.8 \mathrm{~Hz}, 1 \mathrm{H}, 8-\mathrm{H}), 7.73-7.78(\mathrm{~m}, 1 \mathrm{H}, 7-\mathrm{H}), 7.75$ (d, $J$ $\left.=8.5 \mathrm{~Hz}, 2 \mathrm{H}, 3^{\prime \prime}, 5^{\prime \prime}-\mathrm{H}\right), 8.00(\mathrm{dd}, J=7.7,1.3 \mathrm{~Hz}, 1 \mathrm{H}, 5-\mathrm{H}) \mathrm{ppm}$. ${ }^{13} \mathrm{C}$ NMR [75 MHz, $\left.\left(\mathrm{CD}_{3}\right)_{2} \mathrm{SO}+\mathrm{TFA}\right]: \delta=111.1(\mathrm{C}-3), 118.8(\mathrm{C}-$ 8), 122.8 (C-4' '), 123.5 (C- $\alpha, \mathrm{C}-\beta), 123.9$ (C-4a), 125.1 (C-5), 125.6 (C-6), $128.7\left(\mathrm{C}-1^{\prime \prime}\right), 130.3\left(\mathrm{C}-2^{\prime \prime}, 6^{\prime \prime}\right), 132.5\left(\mathrm{C}-3^{\prime \prime}, 5^{\prime \prime}\right), 134.5$ (C7), 138.2 (C-4'), 142.2 (C-5'), 155.8 (C-8a), 161.2 (C-2), 177.6 (C4) ppm. HRMS-EI: $m / z$ calcd. for $\mathrm{C}_{19} \mathrm{H}_{12} \mathrm{O}_{2} \mathrm{~N}_{3}{ }^{79} \mathrm{Br} \mathrm{M}^{+\cdot} 393.0113$, found 393.0122; calcd. for $\mathrm{C}_{19} \mathrm{H}_{12} \mathrm{O}_{2} \mathrm{~N}_{3}{ }^{81} \mathrm{Br} \mathrm{M} \mathrm{M}^{+\cdot} 395.0092$, found 395.0106. MS $\left(\mathrm{ESI}^{+}\right): m / z(\%)=394(39)[\mathrm{M}+\mathrm{H}]^{+}\left({ }^{79} \mathrm{Br}\right) ;(37) 396$ $[\mathrm{M}+\mathrm{H}]^{+}\left({ }^{81} \mathrm{Br}\right)$.

General Procedure for the Synthesis of $(E)$-2-[2-(4-Aryl-2-methyl2H-1,2,3-triazol-5-yl)vinyl]-4H-chromen-4-ones 9a-d, $(E)$-2-[2-(4Aryl-3-methyl-2H-1,2,3-triazol-5-yl)vinyl]-4H-chromen-4-ones 10ad and $(E)-2-[2-(4-A r y l-1-m e t h y l-2 H-1,2,3-t r i a z o l-5-y l) v i n y l]-4 H-$ chromen-4-ones 11a-d: Dimethyl sulfate $(0.1 \mathrm{~mL}, 1.1 \mathrm{mmol})$ was added dropwise to a solution of the appropriate $(E)$-2-[2-(4-aryl$2 H$-1,2,3-triazol-5-yl)vinyl]-4H-chromen-4-one 8a-d (1 mmol) in acetone $(10 \mathrm{~mL})$ with potassium carbonate $(0.36 \mathrm{~g}, 3 \mathrm{mmol})$. The mixture was stirred under reflux for $1 \mathrm{~h}$. The inorganic salts were removed by filtration and washed with acetone $(3 \times 30 \mathrm{~mL})$. The solvent was evaporated to dryness and the residue was purified by silica column chromatography using $\mathrm{CH}_{2} \mathrm{Cl}_{2}$ as eluent. Two fractions were obtained: 1,2,3-triazole derivatives 9a-d (higher $R_{\mathrm{f}}$ value) in $67-82 \%$ yield and a mixture of $\mathbf{1 0 a - d}$ and 11a-d in 18 $33 \%$ yield.

(E)-2-[2-(2-Methyl-4-phenyl-2H-1,2,3-triazol-5-yl)vinyl]-4Hchromen-4-one (9a): Yield $221 \mathrm{mg}(67 \%)$, m.p. $169-171{ }^{\circ} \mathrm{C} .{ }^{1} \mathrm{H}$ NMR (300 MHz, $\left.\mathrm{CDCl}_{3}\right): \delta=4.29\left(\mathrm{~s}, 3 \mathrm{H}, \mathrm{NCH}_{3}\right), 6.34(\mathrm{~s}, 1 \mathrm{H}$, 3-H), $7.12(\mathrm{~d}, J=15.8 \mathrm{~Hz}, 1 \mathrm{H}, \alpha-\mathrm{H}), 7.39$ (ddd, $J=8.0,7.1$, $0.8 \mathrm{~Hz}, 1 \mathrm{H}, 6-\mathrm{H}), 7.47$ (dd, $J=8.2,0.8 \mathrm{~Hz}, 1 \mathrm{H}, 8-\mathrm{H}), 7.48-7.57$ $\left(\mathrm{m}, 3 \mathrm{H}, 3^{\prime \prime}, 4^{\prime \prime}, 5^{\prime \prime}-\mathrm{H}\right), 7.61(\mathrm{~d}, J=15.8 \mathrm{~Hz}, 1 \mathrm{H}, \beta-\mathrm{H}), 7.64-7.70$ (m, $\left.3 \mathrm{H}, 7-\mathrm{H}, 2^{\prime \prime}, 6^{\prime \prime}-\mathrm{H}\right), 8.19$ (dd, $\left.J=8.0,1.5 \mathrm{~Hz}, 1 \mathrm{H}, 5-\mathrm{H}\right) \mathrm{ppm}$. ${ }^{13} \mathrm{C}$ NMR (75 MHz, $\left.\mathrm{CDCl}_{3}\right): \delta=42.2\left(\mathrm{NCH}_{3}\right), 111.3(\mathrm{C}-3), 117.9$ 
(C-8), 123.2 (C- $\alpha$ ), 124.0 (C- $\beta$ ), 124.1 (C-4a), 125.1 (C-6), 125.7 (C5), $128.3\left(\mathrm{C}-2^{\prime \prime}, 6^{\prime \prime}\right), 128.9$ (C-4" $), 129.0\left(\mathrm{C}-3^{\prime \prime}, 5^{\prime \prime}\right), 130.1\left(\mathrm{C}-1^{\prime \prime}\right)$, 133.8 (C-8), 140.5 (C-5'), 147.4 (C-4'), 155.9 (C-8a), 161.1 (C-2), 178.5 (C-4) ppm. HRMS (ESI ${ }^{+}$): $m / z$ calcd. for $\mathrm{C}_{20} \mathrm{H}_{16} \mathrm{~N}_{3} \mathrm{O}_{2}[\mathrm{M}+$ $\mathrm{H}]^{+}$330.1243, found 330.1231. MS $\left(\mathrm{ESI}^{+}\right): \mathrm{m} / z(\%)=330(100)$ $[2 \mathrm{M}+\mathrm{H}]^{+}, 659(7)[2 \mathrm{M}+\mathrm{H}]^{+}$

Mixture of 10a and 11a (86:14): Yield $109 \mathrm{mg}(33 \%)$. (E)-2-[2-(3Methyl-4-phenyl-1 $\boldsymbol{H}$-1,2,3-triazol-5-yl)vinyl]-4 $\boldsymbol{H}$-chromen-4-one (10a): ${ }^{1} \mathrm{H}$ NMR $\left(500 \mathrm{MHz}, \mathrm{CDCl}_{3}\right): \delta=4.02\left(\mathrm{~s}, 3 \mathrm{H}, \mathrm{NCH}_{3}\right), 6.30$ (s, $1 \mathrm{H}, 3-\mathrm{H}), 7.21(\mathrm{~d}, J=15.8 \mathrm{~Hz}, 1 \mathrm{H}, \alpha-\mathrm{H}), 7.36(\mathrm{~d}, J=15.8 \mathrm{~Hz}$, $1 \mathrm{H}, \beta-\mathrm{H}), 7.38$ (ddd, $J=7.7,7.5,1.0 \mathrm{~Hz}, 1 \mathrm{H}, 6-\mathrm{H}), 7.41-7.43$ (m, $\left.3 \mathrm{H}, 8-\mathrm{H}, 2^{\prime \prime}, 6^{\prime \prime}-\mathrm{H}\right), 7.61-7.65\left(\mathrm{~m}, 4 \mathrm{H}, 7-\mathrm{H}, 3^{\prime \prime}, 4^{\prime \prime}, 5^{\prime \prime}-\mathrm{H}\right), 8.17$ (dd, $J=7.5,1.5 \mathrm{~Hz}, 1 \mathrm{H}, 5-\mathrm{H}) \mathrm{ppm} .{ }^{13} \mathrm{C} \mathrm{NMR}\left(75 \mathrm{MHz}, \mathrm{CDCl}_{3}\right)$; $\delta=35.5\left(\mathrm{NCH}_{3}\right), 110.9(\mathrm{C}-3), 117.9(\mathrm{C}-8), 122.0(\mathrm{C}-\alpha), 124.0(\mathrm{C}-$ B), 124.1 (C-4a), 125.0 (C-6), 125.7 (C-5), 126.1 (C-1"'), 129.5 (C$\left.2^{\prime \prime}, 6^{\prime \prime}\right), 129.6\left(\mathrm{C}-3^{\prime \prime}, 5^{\prime \prime}\right), 130.2\left(\mathrm{C}-4^{\prime \prime}\right), 133.6$ (C-7), $137.0\left(\mathrm{C}-4^{\prime}\right)$, 141.3 (C-5'), 155.9 (C-8a), 161.4 (C-2), 178.5 (C-4) ppm. (E)-2-[2(1-Methyl-4-phenyl-1 H-1,2,3-triazol-5-yl)vinyl]-4H-chromen-4-one (11a): ${ }^{1} \mathrm{H}$ NMR $\left(500 \mathrm{MHz}, \mathrm{CDCl}_{3}\right.$ ): $\delta=4.27$ (s, $3 \mathrm{H}, \mathrm{NCH}_{3}$ ), 6.27 (s, $1 \mathrm{H}, 3-\mathrm{H}) \mathrm{ppm}$.

(E)-2-\{2-[2-Methyl-4-(4-methylphenyl)-2H-1,2,3-triazol-5-yl]vinyl\}4H-chromen-4-one (9b): Yield $257 \mathrm{mg}(75 \%)$, m.p. $192-194{ }^{\circ} \mathrm{C} .{ }^{1} \mathrm{H}$ NMR $\left(300 \mathrm{MHz}, \mathrm{CDCl}_{3}\right): \delta=2.46\left(\mathrm{~s}, 3 \mathrm{H}, \mathrm{CH}_{3}\right), 4.29(\mathrm{~s}, 3 \mathrm{H}, \mathrm{N}-$ $\left.\mathrm{CH}_{3}\right), 6.49$ (s, $\left.1 \mathrm{H}, 3-\mathrm{H}\right), 7.13,(\mathrm{~d}, J=15.8 \mathrm{~Hz}, 1 \mathrm{H}, \alpha-\mathrm{H}), 7.34$ (d, $\left.J=8.0 \mathrm{~Hz}, 2 \mathrm{H}, 3^{\prime \prime}, 5^{\prime \prime}-\mathrm{H}\right), 7.42(\mathrm{ddd}, J=7.7,7.4,1.1 \mathrm{~Hz}, 1 \mathrm{H}, 6-$ $\mathrm{H}), 7.50(\mathrm{dd}, J=8.4,1.1 \mathrm{~Hz}, 1 \mathrm{H}, 8-\mathrm{H}), 7.55(\mathrm{~d}, J=8.0 \mathrm{~Hz}, 2 \mathrm{H}$, $\left.2^{\prime \prime}, 6^{\prime \prime}-\mathrm{H}\right), 7.64(\mathrm{~d}, J=15.8 \mathrm{~Hz}, 1 \mathrm{H}, \beta-\mathrm{H}), 7.70(\mathrm{ddd}, J=8.4,7.4$, $1.6 \mathrm{~Hz}, 1 \mathrm{H}, 7-\mathrm{H}), 8.21(\mathrm{dd}, J=7.7,1.6 \mathrm{~Hz}, 1 \mathrm{H}, 5-\mathrm{H}) \mathrm{ppm} .{ }^{13} \mathrm{C}$ NMR $\left(75 \mathrm{MHz}, \mathrm{CDCl}_{3}\right): \delta=21.4\left(\mathrm{CH}_{3}\right), 42.2\left(\mathrm{NCH}_{3}\right), 110.8(\mathrm{C}-$ 3), 118.0 (C-8), 122.7 (C- $\alpha$ ), 123.6 (C-4a), 124.9 (C- $\beta), 125.3$ (C-6), 125.7 (C-5), $127.1\left(\mathrm{C}-1^{\prime \prime}\right), 128.2\left(\mathrm{C}-2^{\prime \prime}, 6^{\prime \prime}\right), 129.8\left(\mathrm{C}-3^{\prime \prime}, 5^{\prime \prime}\right), 134.1$ (C-7), 139.1 (C-4'), 140.3 (C-5'), 147.7 (C-4' $), 156.0$ (C-8a), 161.9 (C-2), 178.5 (C-4) ppm. HRMS (ESI $\left.{ }^{+}\right): m / z$ calcd. for $\mathrm{C}_{21} \mathrm{H}_{18} \mathrm{~N}_{3} \mathrm{O}_{2}$ $[\mathrm{M}+\mathrm{H}]^{+}$344.1399, found 344.1381. MS $\left(\mathrm{ESI}^{+}\right): m / z(\%)=344$ (100) $[\mathrm{M}+\mathrm{H}]^{+}, 687(11)[2 \mathrm{M}+\mathrm{H}]^{+}$

Mixture of 10b and 11b (81:19): Yield $86 \mathrm{mg}(25 \%)$. (E)-2-\{2-[3Methyl-4-(4-methylphenyl)-1 $H$-1,2,3-triazol-5-yl]vinyl $\}-4 H$ chromen-4-one (10b): ${ }^{1} \mathrm{H}$ NMR $\left(300 \mathrm{MHz}, \mathrm{CDCl}_{3}\right): \delta=2.51$ (s, 3 $\left.\mathrm{H}, \mathrm{CH}_{3}\right), 4.01\left(\mathrm{~s}, 3 \mathrm{H}, \mathrm{NCH}_{3}\right), 6.31(\mathrm{~s}, 1 \mathrm{H}, 3-\mathrm{H}), 7.20(\mathrm{~d}, J=$ $15.9 \mathrm{~Hz}, 1 \mathrm{H}, \alpha-\mathrm{H}), 7.30\left(\mathrm{~d}, J=8.1 \mathrm{~Hz}, 2 \mathrm{H}, 2^{\prime \prime}, 6^{\prime \prime}-\mathrm{H}\right), 7.32-7.38$ $(\mathrm{m}, 1 \mathrm{H}, 6-\mathrm{H}), 7.37(\mathrm{~d}, J=15.9 \mathrm{~Hz}, 1 \mathrm{H}, \beta-\mathrm{H}), 7.41-7.45(\mathrm{~m}, 3 \mathrm{H}$, $8-\mathrm{H}, 3^{\prime \prime}, 5^{\prime \prime}-\mathrm{H}$ ), 7.64 (ddd, $J=8.5,7.1,1.6 \mathrm{~Hz}, 1 \mathrm{H}, 7-\mathrm{H}$ ), 8.17 (dd, $J=7.9,1.6 \mathrm{~Hz}, 1 \mathrm{H}, 5-\mathrm{H}) \mathrm{ppm} .{ }^{13} \mathrm{C} \mathrm{NMR}\left(75 \mathrm{MHz}, \mathrm{CDCl}_{3}\right): \delta=$ $21.5\left(\mathrm{CH}_{3}\right), 35.5\left(\mathrm{NCH}_{3}\right), 110.8(\mathrm{C}-3), 117.9(\mathrm{C}-8), 121.8(\mathrm{C}-\alpha)$, 123.0 (C-4a), $124.0\left(\mathrm{C}-1^{\prime \prime}\right), 124.3$ (C- $\left.\beta\right), 125.0$ (C-6), 125.7 (C-5), $129.4\left(\mathrm{C}-2^{\prime \prime}, 6^{\prime \prime}\right), 130.2\left(\mathrm{C}-3^{\prime \prime}, 5^{\prime \prime}\right), 133.7$ (C-7), 137.2 (C-4'), 140.6 (C-4" ), 141.1 (C-5'), 155.9 (C-8a), 161.6 (C-2), 178.5 (C-4) ppm. (E)-2-\{2-[1-Methyl-4-(4-methylphenyl)-1 H-1,2,3-triazol-5-yl]vinyl\}4H-chromen-4-one (11b): ${ }^{1} \mathrm{H}$ NMR $\left(300 \mathrm{MHz}, \mathrm{CDCl}_{3}\right): \delta=2.2(\mathrm{~s}$, $\left.3 \mathrm{H}, \mathrm{CH}_{3}\right), 3.97$ (s, $\left.3 \mathrm{H}, \mathrm{NCH}_{3}\right), 6.40$ (s, $\left.1 \mathrm{H}, 3-\mathrm{H}\right)$ ppm.

(E)-2-\{2-[4-(4-Methoxyphenyl)-2-methyl-2 H-1,2,3-triazol-5yl|vinyl\}-4H-chromen-4-one (9c): Yield $280 \mathrm{mg}$ (78\%), m.p. 186 $187^{\circ} \mathrm{C} .{ }^{1} \mathrm{H}$ NMR $\left(300 \mathrm{MHz}, \mathrm{CDCl}_{3}\right): \delta=3.90\left(\mathrm{~s}, 3 \mathrm{H}, \mathrm{OCH}_{3}\right)$, 4.28 (s, $\left.3 \mathrm{H}, \mathrm{NCH}_{3}\right), 6.34(\mathrm{~s}, 1 \mathrm{H}, 3-\mathrm{H}), 7.06(\mathrm{~d}, J=8.8 \mathrm{~Hz}, 2 \mathrm{H}$, $\left.3^{\prime \prime}, 5^{\prime \prime}-\mathrm{H}\right), 7.10(\mathrm{~d}, J=15.8 \mathrm{~Hz}, 1 \mathrm{H}, \alpha-\mathrm{H}), 7.40(\mathrm{ddd}, J=7.7,7.3$, $1.1 \mathrm{~Hz}, 1 \mathrm{H}, 6-\mathrm{H}), 7.48$ (d, $J=8.2 \mathrm{~Hz}, 1 \mathrm{H}, 8-\mathrm{H}), 7.59$ (d, $J=$ $15.8 \mathrm{~Hz}, 1 \mathrm{H}, \beta-\mathrm{H}), 7.59$ (d, $J=8.8 \mathrm{~Hz}, 2 \mathrm{H}, 2^{\prime \prime}, 6^{\prime \prime}-\mathrm{H}$ ), 7.67 (ddd, $J=8.2,7.3,1.6 \mathrm{~Hz}, 1 \mathrm{H}, 7-\mathrm{H}), 8.20(\mathrm{dd}, J=7.7,1.6 \mathrm{~Hz}, 1 \mathrm{H}, 5-$ $\mathrm{H}) \mathrm{ppm} .{ }^{13} \mathrm{C} \mathrm{NMR}\left(75 \mathrm{MHz}, \mathrm{CDCl}_{3}\right): \delta=42.1\left(\mathrm{NCH}_{3}\right), 55.4$ $\left(\mathrm{OCH}_{3}\right), 111.1(\mathrm{C}-3), 114.4\left(\mathrm{C}-3^{\prime \prime}, 5^{\prime \prime}\right), 117.9(\mathrm{C}-8), 122.5\left(\mathrm{C}-1^{\prime \prime}\right)$, 122.9 (C- $\alpha), 124.1$ (C-4a), 124.2 (C- $\beta), 125.0$ (C-6), 125.7 (C-5), $129.6\left(\mathrm{C}-2^{\prime \prime}, 6^{\prime \prime}\right), 133.7(\mathrm{C}-7), 140.2\left(\mathrm{C}-5^{\prime}\right), 147.3\left(\mathrm{C}-4^{\prime}\right), 155.9(\mathrm{C}-$ 8a), 160.2 (C-4' ), 161.1 (C-2), 178.5 (C-4) ppm. HRMS (ESI ${ }^{+}$): $\mathrm{m} / z$ calcd. for $\mathrm{C}_{21} \mathrm{H}_{18} \mathrm{~N}_{3} \mathrm{O}_{3}[\mathrm{M}+\mathrm{H}]^{+} 360.1348$, found 360.1332 . MS $\left(\mathrm{ESI}^{+}\right): m / z(\%)=344(4)\left[\mathrm{M}-\mathrm{CH}_{3}\right]^{+}, 360(100)[\mathrm{M}+\mathrm{H}]^{+}$, $719(10)[2 \mathrm{M}+\mathrm{H}]^{+}$.

Mixture of 10c and 11c (87:13): Yield $79 \mathrm{mg}(22 \%)$. (E)-2-\{2-[4-(4Methoxyphenyl)-3-methyl-1 $H$-1,2,3-triazol-5-yl|vinyl $\}-4 H$-chromen4-one (10c): ${ }^{1} \mathrm{H}$ NMR $\left(300 \mathrm{MHz}, \mathrm{CDCl}_{3}\right): \delta=3.94\left(\mathrm{~s}, 3 \mathrm{H}, \mathrm{OCH}_{3}\right)$, $4.00\left(\mathrm{~s}, 3 \mathrm{H}, \mathrm{NCH}_{3}\right), 6.30(\mathrm{~s}, 1 \mathrm{H}, 3-\mathrm{H}), 7.13(\mathrm{~d}, J=8.8 \mathrm{~Hz}, 2 \mathrm{H}$, $\left.3^{\prime \prime}, 5^{\prime \prime}-\mathrm{H}\right), 7.19(\mathrm{~d}, J=15.9 \mathrm{~Hz}, 1 \mathrm{H}, \alpha-\mathrm{H}), 7.33-7.40(\mathrm{~m}, 4 \mathrm{H}, \beta-$ $\left.\mathrm{H}, 6-\mathrm{H}, 2^{\prime \prime}, 6^{\prime \prime}-\mathrm{H}\right), 7.43(\mathrm{dd}, J=8.5,0.6 \mathrm{~Hz}, 1 \mathrm{H}, 8-\mathrm{H}), 7.64$ (ddd, $J=8.5,7.0,1.6 \mathrm{~Hz}, 1 \mathrm{H}, 7-\mathrm{H}), 8.16(\mathrm{dd}, J=7.9,1.6 \mathrm{~Hz}, 1 \mathrm{H}, 5-$ $\mathrm{H}) \mathrm{ppm} .{ }^{13} \mathrm{C} \mathrm{NMR}\left(75 \mathrm{MHz}, \mathrm{CDCl}_{3}\right): \delta=35.4\left(\mathrm{NCH}_{3}\right), 55.5$ $\left(\mathrm{OCH}_{3}\right), 110.8(\mathrm{C}-3), 115.0\left(\mathrm{C}-3^{\prime \prime}, 5^{\prime \prime}\right), 117.9(\mathrm{C}-8), 121.6(\mathrm{C}-\alpha)$, 124.1 (C-1"'), 124.3 (C- $\beta$ ), 125.0 (C-6), 125.7 (C-5), 130.9 (C$\left.2^{\prime \prime}, 6^{\prime \prime}\right), 133.6$ (C-7), 137.0 (C-4'), 141.2 (C-5'), 155.9 (C-8a), 161.0 $\left(\mathrm{C}-4^{\prime \prime}\right), 161.5$ (C-2), 178.5 (C-4) ppm. (E)-2-\{2-[4-(4-Methoxyphenyl)-1-methyl-1 $\boldsymbol{H}$-1,2,3-triazol-5-yl|vinyl $\}$-4H-chromen-4-one (11c): ${ }^{1} \mathrm{H}$ NMR (300 MHz, $\left.\mathrm{CDCl}_{3}\right): \delta=3.88\left(\mathrm{~s}, 3 \mathrm{H}, \mathrm{OCH}_{3}\right), 4.25$ (s, $3 \mathrm{H}, \mathrm{NCH}_{3}$ ), 6.27 (s, $\left.1 \mathrm{H}, 3-\mathrm{H}\right) \mathrm{ppm}$.

(E)-2-\{2-[4-(4-Bromophenyl)-2-methyl-2H-1,2,3-triazol-5-yl]vinyl\}4H-chromen-4-one (9d): Yield $335 \mathrm{mg}(82 \%)$, m.p. $223-224{ }^{\circ} \mathrm{C} .{ }^{1} \mathrm{H}$ NMR (300 MHz, $\left.\mathrm{CDCl}_{3}\right): \delta=4.29\left(\mathrm{~s}, 3 \mathrm{H}, \mathrm{NCH}_{3}\right), 6.50(\mathrm{~s}, 1 \mathrm{H}$, $3-\mathrm{H}), 7.15(\mathrm{~d}, J=15.8 \mathrm{~Hz}, 1 \mathrm{H}, \alpha-\mathrm{H}), 7.43(\mathrm{dt}, J=7.7,0.8 \mathrm{~Hz}, 1$ $\mathrm{H}, 6-\mathrm{H}), 7.52(\mathrm{dd}, J=7.6,0.8 \mathrm{~Hz}, 1 \mathrm{H}, 8-\mathrm{H}), 7.54(\mathrm{~d}, J=8.5 \mathrm{~Hz}$, $\left.2 \mathrm{H}, 2^{\prime \prime}, 6^{\prime \prime}-\mathrm{H}\right), 7.58(\mathrm{~d}, J=15.8 \mathrm{~Hz}, 1 \mathrm{H}, \beta-\mathrm{H}), 7.67(\mathrm{~d}, J=8.5 \mathrm{~Hz}$, $\left.2 \mathrm{H}, 3^{\prime \prime}, 5^{\prime \prime}-\mathrm{H}\right), 7.68-7.74(\mathrm{~m}, 1 \mathrm{H}, 7-\mathrm{H}), 8.21(\mathrm{dd}, J=7.7,1.6 \mathrm{~Hz}$, $1 \mathrm{H}, 5-\mathrm{H}) \mathrm{ppm} .{ }^{13} \mathrm{C} \mathrm{NMR}\left(75 \mathrm{MHz}, \mathrm{CDCl}_{3}\right): \delta=42.2\left(\mathrm{NCH}_{3}\right)$, 111.1 (C-3), 117.9 (C-8), 123.3 (C-4" ), $123.5(\mathrm{C}-\beta), 123.7$ (C- $\alpha)$, 124.1 (C-4a), 125.2 (C-6), 125.7 (C-5), 129.1 (C-1"'), 129.8 (C$\left.2^{\prime \prime}, 6^{\prime \prime}\right), 132.2\left(\mathrm{C}-3^{\prime \prime}, 5^{\prime \prime}\right), 133.9(\mathrm{C}-7), 140.5\left(\mathrm{C}-5^{\prime}\right), 146.3\left(\mathrm{C}-4^{\prime}\right)$, 155.9 (C-8a), 160.9 (C-2), 178.5 (C-4) ppm. HRMS (ESI $\left.{ }^{+}\right): m / z$ calcd. for $\mathrm{C}_{20} \mathrm{H}_{15}{ }^{79} \mathrm{BrN}_{3} \mathrm{O}_{2}[\mathrm{M}+\mathrm{H}]^{+}$408.0348, found 408.0330; calcd. for $\mathrm{C}_{20} \mathrm{H}_{15}{ }^{81} \mathrm{BrN}_{3} \mathrm{O}_{2}[\mathrm{M}+\mathrm{H}]^{+}$410.0327, found 410.0306 . MS $\left(\mathrm{ESI}^{+}\right): m / z(\%)=408(100)[\mathrm{M}+\mathrm{H}]^{+}, 430(15)[\mathrm{M}+\mathrm{Na}]^{+}$.

Mixture of 10d and 11d (93:7): Yield $73 \mathrm{mg}$ (18\%). (E)-2-\{2-[4-(4Bromophenyl)-1-methyl-1 $\boldsymbol{H}$-1,2,3-triazol-5-yl]vinyl\}-4H-chromen-4one (10d): ${ }^{1} \mathrm{H}$ NMR $\left(500 \mathrm{MHz}, \mathrm{CDCl}_{3}\right): \delta=4.02$ (s, $3 \mathrm{H}, \mathrm{NCH}_{3}$ ), $6.33(\mathrm{~s}, 1 \mathrm{H}, 3-\mathrm{H}), 7.26-7.31(\mathrm{~m}, 2 \mathrm{H}, \alpha-\mathrm{H}, \beta-\mathrm{H}), 7.30(\mathrm{~d}, J=$ $\left.8.5 \mathrm{~Hz}, 2 \mathrm{H}, 2^{\prime \prime}, 6^{\prime \prime}-\mathrm{H}\right), 7.38$ (ddd, $\left.J=7.9,7.1,0.7 \mathrm{~Hz}, 1 \mathrm{H}, 6-\mathrm{H}\right)$, 7.45 (dd, $J=8.5,0.7 \mathrm{~Hz}, 1 \mathrm{H}, 8-\mathrm{H}), 7.65(\mathrm{ddd}, J=8.5,7.1,1.6 \mathrm{~Hz}$, $1 \mathrm{H}, 7-\mathrm{H}), 7.77\left(\mathrm{~d}, J=8.5 \mathrm{~Hz}, 2 \mathrm{H}, 3^{\prime \prime}, 5^{\prime \prime}-\mathrm{H}\right), 8.18$ (dd, $J=7.9$, $1.6 \mathrm{~Hz}, 1 \mathrm{H}, 5-\mathrm{H}) \mathrm{ppm} .{ }^{13} \mathrm{C} \mathrm{NMR}\left(125 \mathrm{MHz}, \mathrm{CDCl}_{3}\right): \delta=35.5$ $\left(\mathrm{NCH}_{3}\right), 111.2(\mathrm{C}-3), 117.9(\mathrm{C}-8), 122.5(\mathrm{C}-\beta), 123.3(\mathrm{C}-\alpha), 124.1$ (C-4a), 124.9 and 125.0 (C-1" and C-4"'), 125.1 (C-6), 125.7 (C-5), 131.1 (C-2"',6" ), 132.9 (C-3" ,5" ), 133.7 (C-7), 135.9 (C-4'), 141.5 (C-5'), 155.9 (C-8a), 161.2 (C-2), 178.4 (C-4) ppm. (E)-2-\{2-[4-(4Bromophenyl)-3-methyl-1 $\boldsymbol{H}$-1,2,3-triazol-5-yl|vinyl\}-4H-chromen-4one (11d): ${ }^{1} \mathrm{H}$ NMR $\left(500 \mathrm{MHz}, \mathrm{CDCl}_{3}\right): \delta=4.03$ (s, $3 \mathrm{H}, \mathrm{NCH}_{3}$ ), $6.30(\mathrm{~s}, 1 \mathrm{H}, 3-\mathrm{H}) \mathrm{ppm}$.

General Procedure for the Synthesis of 4-(4-Aryl-2-methyl-2H-1,2,3triazol-5-yl)-2-methyl-3a,4,5,11b-tetrahydrochromeno[3,2-e]isoindole-1,3,11(2H)-triones (12a-d). Method A: $N$-Methylmaleimide $(0.10 \mathrm{~g}, 0.92 \mathrm{mmol})$ was added to a solution of the appropriate $(E)$ 2-[2-(4-aryl-2-methyl-2 $H$-1,2,3-triazol-5-yl)vinyl]-4 $H$-chromen-4ones 9a-d $(0.18 \mathrm{mmol})$ in dry DMF $(5 \mu \mathrm{L})$. The mixture was heated at $160{ }^{\circ} \mathrm{C}$ under microwave irradiation (multimode apparatus) for $20 \mathrm{~min}$. The residue was dissolved in $\mathrm{CH}_{2} \mathrm{Cl}_{2}$ and purified by preparative TLC using $\mathrm{CH}_{2} \mathrm{Cl}_{2} /$ EtOAc (4:1) as eluent to give desired cycloadducts $\mathbf{1 2 a}-\mathbf{d}$ in the following yields: $\mathbf{1 2 a}(29.3 \mathrm{mg}, 37 \%)$, 12b (27.0 mg, 33\%), 12c (33.9 mg, 40\%), 12d (28.0 mg, 30\%).

Method B: $N$-Methylmaleimide $(0.10 \mathrm{~g}, 0.92 \mathrm{mmol})$ was mixed with the appropriate $(E)-2-[2-(4-a r y l-2-m e t h y l-2 H-1,2,3-$ triazol-5-yl) 
vinyl]-4H-chromen-4-ones $9 \mathbf{9}-\mathbf{d}(0.18 \mathrm{mmol})$ in a close vessel. The mixture was heated at $200{ }^{\circ} \mathrm{C}$ under microwave irradiation (monomode apparatus) for $10 \mathrm{~min}$. The residue was dissolved in $\mathrm{CH}_{2} \mathrm{Cl}_{2}$ and purified by preparative TLC using $\mathrm{CH}_{2} \mathrm{Cl}_{2} /$ EtOAc (4:1) as eluent to give desired cycloadducts 12a-d in the following yields: 12a (28.5 mg, 36\%), 12b (39.3 mg, 48\%), 12c (33.9 mg, $40 \%)$, 12d (35.5 mg, 38\%).

2-Methyl-4-(2-methyl-4-phenyl-2H-1,2,3-triazol-5-yl)-3a,4,5,11btetrahydrochromeno[3,2-e]isoindole-1,3,11(2H)-trione (12a): M.p. $152-153{ }^{\circ} \mathrm{C} .{ }^{1} \mathrm{H}$ NMR $\left(300 \mathrm{MHz}, \mathrm{CDCl}_{3}\right): \delta=2.82(\mathrm{~s}, 3 \mathrm{H}, 2-$ $\left.\mathrm{NCH}_{3}\right), 2.98-3.24(\mathrm{~m}, 2 \mathrm{H}, 5-\mathrm{H}), 3.47$ (dd, $J=8.8,6.4 \mathrm{~Hz}, 1 \mathrm{H}$, 3a-H), 3.84-3.91 (m, $1 \mathrm{H}, 4-\mathrm{H}), 4.18$ (s, $3 \mathrm{H}, 2^{\prime}-\mathrm{NCH}_{3}$ ), 4.64 (d, $J$ $=8.8 \mathrm{~Hz}, 1 \mathrm{H}, 11 \mathrm{~b}-\mathrm{H}), 7.40-7.49\left(\mathrm{~m}, 5 \mathrm{H}, 7-\mathrm{H}, 9-\mathrm{H}, 3^{\prime \prime}, 4^{\prime \prime}, 5^{\prime \prime}-\mathrm{H}\right)$, 7.61 (dd, $\left.J=8.1,1.5 \mathrm{~Hz}, 2 \mathrm{H}, 2^{\prime \prime}, 6^{\prime \prime}-\mathrm{H}\right), 7.67$ (ddd, $J=8.5,7.1$, $1.6 \mathrm{~Hz}, 1 \mathrm{H}, 8-\mathrm{H}), 8.30(\mathrm{dd}, J=8.4,1.6 \mathrm{~Hz}, 1 \mathrm{H}, 10-\mathrm{H}) \mathrm{ppm} .{ }^{13} \mathrm{C}$ NMR (75 MHz, $\left.\mathrm{CDCl}_{3}\right): \delta=24.9\left(2-\mathrm{NCH}_{3}\right), 30.6(\mathrm{C}-5), 32.4(\mathrm{C}-$ 4), 37.9 (C-11b), 41.2 (C-3a), $41.7\left(2^{\prime}-\mathrm{NCH}_{3}\right), 113.3$ (C-11a), 117.7 (C-7), 123.7 (C-10a), 125.3 (C-9), 126.4 (C-10), 127.7 (C-2' , 6" $)$, $128.6\left(\mathrm{C}-4^{\prime \prime}\right), 129.0\left(\mathrm{C}-3^{\prime \prime}, 5^{\prime \prime}\right), 130.9\left(\mathrm{C}-1^{\prime \prime}\right), 133.7$ (C-8), 142.3 (C5'), 145.1 (C-4'), 155.7 (C-6a), 164.1 (C-5a), 174.7 (C-3), 175.2 (C1), 176.1 (C-11) ppm. HRMS (ESI ${ }^{+}$): $\mathrm{m} / z$ calcd. for $\mathrm{C}_{25} \mathrm{H}_{21} \mathrm{~N}_{4} \mathrm{O}_{4}$ $[\mathrm{M}+\mathrm{H}]^{+}$441.1563, found 441.1539. $\mathrm{MS}\left(\mathrm{ESI}^{+}\right): \mathrm{m} / \mathrm{z}(\%)=441$ (100) $[\mathrm{M}+\mathrm{H}]^{+}, 463(17)[\mathrm{M}+\mathrm{Na}]^{+}, 479(10)[\mathrm{M}+\mathrm{K}]^{+}$.

2-Methyl-4-[2-methyl-4-(4-methylphenyl)-2 H-1,2,3-triazol-5-yl]3a,4,5,11b-tetrahydrochromeno[3,2-e]isoindole-1,3,11(2H)-trione (12b): M.p. $143-145{ }^{\circ} \mathrm{C} .{ }^{1} \mathrm{H}$ NMR $\left(300 \mathrm{MHz}, \mathrm{CDCl}_{3}\right): \delta=2.40$ (s, $\left.3 \mathrm{H}, \mathrm{CH}_{3}\right), 2.82\left(\mathrm{~s}, 3 \mathrm{H}, 2-\mathrm{NCH}_{3}\right), 2.98-3.24(\mathrm{~m}, 2 \mathrm{H}, 5-\mathrm{H}), 3.48$ (dd, $J=8.8,6.4 \mathrm{~Hz}, 1 \mathrm{H}, 3 \mathrm{a}-\mathrm{H}), 3.82-3.89$ (m, $1 \mathrm{H}, 4-\mathrm{H}), 4.17$ (s, $\left.3 \mathrm{H}, 2^{\prime}-\mathrm{NCH}_{3}\right), 4.63(\mathrm{~d}, J=8.8 \mathrm{~Hz}, 1 \mathrm{H}, 11 \mathrm{~b}-\mathrm{H}), 7.26(\mathrm{~d}, J=$ $\left.8.0 \mathrm{~Hz}, 2 \mathrm{H}, 3^{\prime \prime}, 5^{\prime \prime}-\mathrm{H}\right), 7.40-7.45(\mathrm{~m}, 1 \mathrm{H}, 9-\mathrm{H}), 7.41$ (d, $J=$ $8.2 \mathrm{~Hz}, 1 \mathrm{H}, 7-\mathrm{H}), 7.49$ (d, $J=8.0 \mathrm{~Hz}, 2 \mathrm{H}, 2^{\prime \prime}, 6^{\prime \prime}-\mathrm{H}$ ), 7.67 (ddd, $J=8.2,7.2,1.5 \mathrm{~Hz}, 1 \mathrm{H}, 8-\mathrm{H}), 8.29$ (dd, $J=8.2,1.5 \mathrm{~Hz}, 1 \mathrm{H}, 10-$ $\mathrm{H}) \mathrm{ppm} .{ }^{13} \mathrm{C}$ NMR $\left(75 \mathrm{MHz}, \mathrm{CDCl}_{3}\right): \delta=21.3\left(\mathrm{CH}_{3}\right), 24.9(2-$ $\mathrm{NCH}_{3}$ ), 30.5 (C-5), 32.4 (C-4), 37.9 (C-11b), 41.1 (C-3a), 41.7 (2' $\mathrm{NCH}_{3}$ ), 113.3 (C-11a), 117.7 (C-7), 123.7 (C-10a), 125.3 (C-9), 126.4 (C-10), $127.5\left(\mathrm{C}-2^{\prime \prime}, 6^{\prime \prime}\right), 127.9\left(\mathrm{C}-1^{\prime \prime}\right), 129.7\left(\mathrm{C}-3^{\prime \prime}, 5^{\prime \prime}\right)$, 133.7 (C-8), 138.6 (C-4'), 142.2 (C-5'), 145.1 (C-4'), 155.7 (C-6a), 164.2 (C-5a), 174.8 (C-3), 175.2 (C-1), 176.0 (C-11) ppm. HRMS $\left(\mathrm{ESI}^{+}\right): \mathrm{m} / z$ calcd. for $\mathrm{C}_{26} \mathrm{H}_{23} \mathrm{~N}_{4} \mathrm{O}_{4}[\mathrm{M}+\mathrm{H}]^{+} 455.1719$, found 455.1699. MS $\left(\mathrm{ESI}^{+}\right): m / z(\%)=455(100)[\mathrm{M}+\mathrm{H}]^{+}, 477(26)[\mathrm{M}$ $+\mathrm{Na}]^{+}$.

4-[4-(4-Methoxyphenyl)-2-methyl-2H-1,2,3-triazol-5-yl]-2-methyl3a,4,5,11b-tetrahydrochromeno[3,2-e]isoindole-1,3,11(2H)-trione (12c): M.p. $167-169^{\circ} \mathrm{C} .{ }^{1} \mathrm{H}$ NMR $\left(300 \mathrm{MHz}, \mathrm{CDCl}_{3}\right): \delta=2.82$ (s, $\left.3 \mathrm{H}, 2-\mathrm{NCH}_{3}\right), 2.97-3.23(\mathrm{~m}, 2 \mathrm{H}, 5-\mathrm{H}), 3.48(\mathrm{dd}, J=8.8,6.4 \mathrm{~Hz}$, $1 \mathrm{H}, 3 \mathrm{a}-\mathrm{H}), 3.79-3.90(\mathrm{~m}, 1 \mathrm{H}, 4-\mathrm{H}), 3.85\left(\mathrm{~s}, 3 \mathrm{H}, \mathrm{OCH}_{3}\right), 4.16(\mathrm{~s}$, $\left.3 \mathrm{H}, 2^{\prime}-\mathrm{NCH}_{3}\right), 4.64(\mathrm{~d}, J=8.8 \mathrm{~Hz}, 1 \mathrm{H}, 11 \mathrm{~b}-\mathrm{H}), 6.98(\mathrm{~d}, J=$ $\left.8.8 \mathrm{~Hz}, 2 \mathrm{H}, 3^{\prime \prime}, 5^{\prime \prime}-\mathrm{H}\right), 7.41$ (d, $\left.J=8.2 \mathrm{~Hz}, 1 \mathrm{H}, 7-\mathrm{H}\right), 7.40-7.45$ (m, $1 \mathrm{H}, 9-\mathrm{H}), 7.53\left(\mathrm{~d}, J=8.8 \mathrm{~Hz}, 2 \mathrm{H}, 2^{\prime \prime}, 6^{\prime \prime}-\mathrm{H}\right), 7.67$ (ddd, $J=$ $8.2,7.1,1.6 \mathrm{~Hz}, 1 \mathrm{H}, 8-\mathrm{H}), 8.29$ (dd, $J=8.4,1.6 \mathrm{~Hz}, 1 \mathrm{H}, 10-$ H) ppm. ${ }^{13} \mathrm{C} \mathrm{NMR}\left(75 \mathrm{MHz}, \mathrm{CDCl}_{3}\right): \delta=24.9\left(2-\mathrm{NCH}_{3}\right), 30.6$ (C-5), 32.4 (C-4), 37.9 (C-11b), 41.2 (C-3a), $41.7\left(2^{\prime}-\mathrm{NCH}_{3}\right), 55.3$ $\left(\mathrm{OCH}_{3}\right), 113.3$ (C-11a), $114.4\left(\mathrm{C}-3^{\prime \prime}, 5^{\prime \prime}\right), 117.7$ (C-7), $123.2\left(\mathrm{C}-1^{\prime \prime}\right)$, 123.6 (C-10a), 125.3 (C-9), 126.4 (C-10), 129.0 (C-2"',6"'), 133.7 (C-8), 142.0 (C-4'), 145.0 (C-5'), 155.7 (C-6a), 159.9 (C-4' $\left.{ }^{\prime \prime}\right), 164.1$ (C-5a), 174.7 (C-3), 175.2 (C-1), 176.0 (C-11) ppm. HRMS (ESI ${ }^{+}$): $\mathrm{m} / \mathrm{z}$ calcd. for $\mathrm{C}_{26} \mathrm{H}_{23} \mathrm{~N}_{4} \mathrm{O}_{5}[\mathrm{M}+\mathrm{H}]^{+} 471.1668$, found 471.1645 . $\mathrm{MS}\left(\mathrm{ESI}^{+}\right): m / z(\%)=471(100)[\mathrm{M}+\mathrm{H}]^{+}, 493(8)[\mathrm{M}+\mathrm{Na}]^{+}$.

4-[4-(4-Bromophenyl)-2-methyl-2H-1,2,3-triazol-5-yl]-2-methyl3a,4,5,11b-tetrahydrochromeno[3,2-e]isoindole-1,3,11(2H)-trione (12d): M.p. $145-146{ }^{\circ} \mathrm{C} .{ }^{1} \mathrm{H}$ NMR $\left(300 \mathrm{MHz}, \mathrm{CDCl}_{3}\right): \delta=2.81$ (s, $\left.3 \mathrm{H}, 2-\mathrm{NCH}_{3}\right), 2.96-3.24(\mathrm{~m}, 2 \mathrm{H}, 5-\mathrm{H}), 3.46(\mathrm{dd}, J=8.8,6.4 \mathrm{~Hz}$,
$1 \mathrm{H}, 3 \mathrm{a}-\mathrm{H}), 3.82-3.88(\mathrm{~m}, 1 \mathrm{H}, 4-\mathrm{H}), 4.16$ (s, $\left.3 \mathrm{H}, 2^{\prime}-\mathrm{NCH}_{3}\right), 4.64$ (d, $J=8.8 \mathrm{~Hz}, 1 \mathrm{H}, 11 \mathrm{~b}-\mathrm{H}), 7.40-7.45(\mathrm{~m}, 1 \mathrm{H}, 9-\mathrm{H}), 7.41$ (d, $J=$ $8.1 \mathrm{~Hz}, 1 \mathrm{H}, 7-\mathrm{H}), 7.49$ (d, $\left.J=8.6 \mathrm{~Hz}, 2 \mathrm{H}, 2^{\prime \prime}, 6^{\prime \prime}-\mathrm{H}\right), 7.59$ (d, $J$ $\left.=8.6 \mathrm{~Hz}, 2 \mathrm{H}, 3^{\prime \prime}, 5^{\prime \prime}-\mathrm{H}\right), 7.67(\mathrm{ddd}, J=8.1,7.2,1.6 \mathrm{~Hz}, 1 \mathrm{H}, 8-$ $\mathrm{H}), 8.29$ (dd, $J=8.2,1.6 \mathrm{~Hz}, 1 \mathrm{H}, 10-\mathrm{H}) \mathrm{ppm} .{ }^{13} \mathrm{C} \mathrm{NMR}(75 \mathrm{MHz}$, $\left.\mathrm{CDCl}_{3}\right): \delta=24.9\left(2-\mathrm{NCH}_{3}\right), 30.7(\mathrm{C}-5), 32.3$ (C-4), 37.9 (C-11b), 41.2 (C-3a), $41.8\left(2^{\prime}-\mathrm{NCH}_{3}\right), 113.3$ (C-11a), 117.7 (C-7), 122.9 (C$\left.4^{\prime \prime}\right), 123.6$ (C-10a), 125.4 (C-9), 126.4 (C-10), 129.3 (C-2'", $\left.6^{\prime \prime}\right)$, $129.8\left(\mathrm{C}-1^{\prime \prime}\right), 132.2\left(\mathrm{C}-3^{\prime \prime}, 5^{\prime \prime}\right), 133.8$ (C-8), $142.4\left(\mathrm{C}-5^{\prime}\right), 144.1$ (C4'), 155.7 (C-6a), 163.8 (C-5a), 174.6 (C-3), 175.2 (C-1), 175.9 (C11) ppm. HRMS $\left(\mathrm{ESI}^{+}\right): \mathrm{m} / z$ calcd. for $\mathrm{C}_{25} \mathrm{H}_{20}{ }^{79} \mathrm{BrN}_{4} \mathrm{O}_{4}[\mathrm{M}+$ $\mathrm{H}]^{+}$519.0668, found 519.0649; calcd. for $\mathrm{C}_{25} \mathrm{H}_{20}{ }^{81} \mathrm{BrN}_{4} \mathrm{O}_{4}[\mathrm{M}+\mathrm{H}]$ +521.0647 , found 521.0627. MS $\left(\mathrm{ESI}^{+}\right): \mathrm{m} / z(\%)=519(20)[\mathrm{M}+$ $\mathrm{H}]^{+}, 541(15)[\mathrm{M}+\mathrm{Na}]^{+}$.

General Procedure for the Synthesis of 4-(5-Aryl-2-methyl-2H-1,2,3triazol-4-yl)-2-methylchromeno[3,2-e $]$ isoindole-1,3,11(2H)-trione (13a-d): 2,3-Dichloro-5,6-dicyanobenzoquinone (DDQ) (121 mg, $534 \mu \mathrm{mol})$ was added to a solution of the appropriate 4-(4-aryl-2methyl-2H-1,2,3-triazol-5-yl)-2-methyl-3a,4,5,11b-tetrahydrochromeno[3,2-e] isoindole-1,3,11(2H)-triones 12a-d $(178 \mu \mathrm{mol})$ in toluene $(10 \mathrm{~mL})$. The mixture was stirred at $100{ }^{\circ} \mathrm{C}$ for $1 \mathrm{~h}$. After that period, the solvent was evaporated to dryness. The residue was purified by preparative TLC using $\mathrm{CH}_{2} \mathrm{Cl}_{2}$ as eluent to give desired 4-(4-aryl-2-methyl-2H-1,2,3-triazol-5-yl)-2-methylchromeno[3,2-e]isoindole-1,3,11(2H)-triones 13a-d in good yields (57-80\%).

2-Methyl-4-(2-methyl-4-phenyl-2 H-1,2,3-triazol-5-yl)chromeno[3,2-e]isoindole-1,3,11(2H)-trione (13a): Yield $57.5 \mathrm{mg}$ (74\%), m.p. $310-312{ }^{\circ} \mathrm{C} .{ }^{1} \mathrm{H} \mathrm{NMR}\left(300 \mathrm{MHz}, \mathrm{CDCl}_{3}\right): \delta=3.07(\mathrm{~s}, 3 \mathrm{H}, 2-$ $\mathrm{NCH}_{3}$ ), 4.35 (s, $\left.3 \mathrm{H}, 2^{\prime}-\mathrm{NCH}_{3}\right), 7.28-7.31$ (m, $\left.3 \mathrm{H}, 3^{\prime \prime}, 4^{\prime \prime}, 5^{\prime \prime}-\mathrm{H}\right)$, 7.39-7.43 (m, $\left.2 \mathrm{H}, 2^{\prime \prime}, 6^{\prime \prime}-\mathrm{H}\right), 7.44-7.49$ (m, $\left.1 \mathrm{H}, 9-\mathrm{H}\right), 7.50$ (d, $J$ $=7.3 \mathrm{~Hz}, 1 \mathrm{H}, 7-\mathrm{H}), 7.78(\mathrm{ddd}, J=8.6,7.3,1.6 \mathrm{~Hz}, 1 \mathrm{H}, 8-\mathrm{H})$, 7.85 (s, $1 \mathrm{H}, 5-\mathrm{H}), 8.40(\mathrm{dd}, J=8.0,1.6 \mathrm{~Hz}, 1 \mathrm{H}, 10-\mathrm{H}) \mathrm{ppm} .{ }^{13} \mathrm{C}$ NMR $\left(75 \mathrm{MHz}, \mathrm{CDCl}_{3}\right): \delta=24.4\left(2-\mathrm{NCH}_{3}\right), 42.1\left(2^{\prime}-\mathrm{NCH}_{3}\right)$, 117.6 (C-7), 119.7 (C-11a), 123.0 (C-10a), 125.2 (C-9), 126.3 (C-5), 127.0 (C-2" $\left.{ }^{\prime \prime} 6^{\prime \prime}\right), 127.4$ (C-3a), 127.5 (C-10), 128.5 (C-4"'), 128.8 $\left(\mathrm{C}-3^{\prime \prime}, 5^{\prime \prime}\right), 130.3\left(\mathrm{C}-1^{\prime \prime}\right), 134.1$ and 134.2 (C-4 and C-11b), 135.5 (C-8), 138.7 (C-5'), 146.5 (C-4'), 155.0 (C-6a), 159.7 (C-5a), 164.4 and $165.4(\mathrm{C}-1$ and $\mathrm{C}-3), 174.3(\mathrm{C}-11) \mathrm{ppm}$. HRMS $\left(\mathrm{ESI}^{+}\right): \mathrm{m} / \mathrm{z}$ calcd. for $\mathrm{C}_{25} \mathrm{H}_{17} \mathrm{~N}_{4} \mathrm{O}_{4}[\mathrm{M}+\mathrm{H}]^{+}$437.1250, found 437.1229. MS $\left(\mathrm{ESI}^{+}\right): m / z(\%)=895(100)[\mathrm{M}+\mathrm{Na}]^{+}, 437(88)[\mathrm{M}+\mathrm{H}]^{+}, 911$ (35) $[2 \mathrm{M}+\mathrm{K}]^{+}$.

2-Methyl-4-[2-methyl-4-(4-methylphenyl)-2H-1,2,3-triazol-5-yl]chromeno[3,2-e]isoindole-1,3,11(2H)-trione (13b): Yield $64.1 \mathrm{mg}$ (80\%), m.p. $302-303{ }^{\circ} \mathrm{C} .{ }^{1} \mathrm{H}$ NMR $\left(300 \mathrm{MHz}, \mathrm{CDCl}_{3}\right): \delta=2.31(\mathrm{~s}$, $\left.3 \mathrm{H}, \mathrm{CH}_{3}\right), 3.08$ (s, $\left.3 \mathrm{H}, 2-\mathrm{NCH}_{3}\right), 4.34$ (s, $\left.3 \mathrm{H}, 2^{\prime}-\mathrm{NCH}_{3}\right), 7.09$ (d, $\left.J=8.1 \mathrm{~Hz}, 2 \mathrm{H}, 3^{\prime \prime}, 5^{\prime \prime}-\mathrm{H}\right), 7.29$ (d, $\left.J=8.1 \mathrm{~Hz}, 2 \mathrm{H}, 2^{\prime \prime}, 6^{\prime \prime}-\mathrm{H}\right)$, 7.44-7.49 (m, $1 \mathrm{H}, 9-\mathrm{H}), 7.50$ (d, $J=7.3 \mathrm{~Hz}, 1 \mathrm{H}, 7-\mathrm{H}), 7.78$ (ddd, $J=8.5,7.3,1.6 \mathrm{~Hz}, 1 \mathrm{H}, 8-\mathrm{H}), 7.83(\mathrm{~s}, 1 \mathrm{H}, 5-\mathrm{H}), 8.39$ (dd, $J=$ 8.0, $1.6 \mathrm{~Hz}, 1 \mathrm{H}, 10-\mathrm{H}) \mathrm{ppm} .{ }^{13} \mathrm{C} \mathrm{NMR}\left(126 \mathrm{MHz}, \mathrm{CDCl}_{3}\right): \delta=$ $21.3\left(\mathrm{CH}_{3}\right), 24.4\left(2-\mathrm{NCH}_{3}\right), 42.1\left(2^{\prime}-\mathrm{NCH}_{3}\right), 117.6(\mathrm{C}-7), 119.7(\mathrm{C}-$ 11a), 123.0 (C-10a), 125.2 (C-9), 126.4 (C-5), 126.9 (C-2'", 6" ), 127.4 (C-3a), $127.5(\mathrm{C}-10), 129.5\left(\mathrm{C}-3^{\prime \prime}, 5^{\prime \prime}\right), 134.1$ and $134.4(\mathrm{C}-$ $11 \mathrm{~b}$ and C-4), 135.5 (C-8), 138.4 (C-5'), 138.5 (C-1" , C-4' $), 146.5$ (C-4'), 155.0 (C-6a), 159.7 (C-5a), 164.3 and 165.5 (C-1 and C-3), $174.2(\mathrm{C}-11) \mathrm{ppm}$. HRMS $\left(\mathrm{ESI}^{+}\right): \mathrm{m} / z$ calcd. for $\mathrm{C}_{26} \mathrm{H}_{19} \mathrm{~N}_{4} \mathrm{O}_{4}[\mathrm{M}$ $+\mathrm{H}]^{+}$451.1406, found 451.1382. MS $\left(\mathrm{ESI}^{+}\right): \mathrm{m} / z(\%)=451(100)$ $[\mathrm{M}+\mathrm{H}]^{+}, 489(63)[\mathrm{M}+\mathrm{K}]^{+}, 923(16)[2 \mathrm{M}+\mathrm{Na}]^{+}, 939(20)[2 \mathrm{M}$ $+\mathrm{K}]^{+}$.

4-[4-(4-Methoxyphenyl)-2-methyl-2H-1,2,3-triazol-5-yl]-2-methylchromeno[3,2-e]isoindole-1,3,11(2H)-trione (13c): Yield $68.9 \mathrm{mg}$ (83\%), m.p. $286-288{ }^{\circ} \mathrm{C} .{ }^{1} \mathrm{H}$ NMR $\left(500 \mathrm{MHz}, \mathrm{CDCl}_{3}\right): \delta=3.09$ (s, $\left.3 \mathrm{H}, 2-\mathrm{NCH}_{3}\right), 3.78\left(\mathrm{~s}, 3 \mathrm{H}, \mathrm{OCH}_{3}\right), 4.33$ (s, $\left.3 \mathrm{H}, 2^{\prime}-\mathrm{NCH}_{3}\right), 6.81$ 
(d, $\left.J=8.9 \mathrm{~Hz}, 2 \mathrm{H}, 3^{\prime \prime}, 5^{\prime \prime}-\mathrm{H}\right), 7.34$ (d, $\left.J=8.9 \mathrm{~Hz}, 2 \mathrm{H}, 2^{\prime \prime}, 6^{\prime \prime}-\mathrm{H}\right)$, 7.46 (ddd, $J=8.0,7.1,0.7 \mathrm{~Hz}, 1 \mathrm{H}, 9-\mathrm{H}), 7.50(\mathrm{dd}, J=8.3,0.7 \mathrm{~Hz}$, $1 \mathrm{H}, 7-\mathrm{H}), 7.78$ (ddd, $J=8.3,7.1,1.6 \mathrm{~Hz}, 1 \mathrm{H}, 8-\mathrm{H}), 7.84$ (s, $1 \mathrm{H}$, $5-\mathrm{H}), 8.40(\mathrm{dd}, J=8.0,1.6 \mathrm{~Hz}, 1 \mathrm{H}, 10-\mathrm{H}) \mathrm{ppm} .{ }^{13} \mathrm{C} \mathrm{NMR}$ $\left(126 \mathrm{MHz}, \mathrm{CDCl}_{3}\right): \delta=24.4\left(2-\mathrm{NCH}_{3}\right), 42.1\left(2^{\prime}-\mathrm{NCH}_{3}\right), 55.3$ $\left(\mathrm{OCH}_{3}\right), 114.3\left(\mathrm{C}-3^{\prime \prime}, 5^{\prime \prime}\right), 117.6(\mathrm{C}-7), 119.6(\mathrm{C}-11 \mathrm{a}), 122.8\left(\mathrm{C}-1^{\prime \prime}\right)$, 123.0 (C-10a), 125.2 (C-9), 126.4 (C-5), 127.4 (C-3a), 127.5 (C-10), $128.3\left(\mathrm{C}-2^{\prime \prime}, 6^{\prime \prime}\right), 134.1$ and $134.4(\mathrm{C}-4$ and $\mathrm{C}-11 \mathrm{~b}), 135.5(\mathrm{C}-8)$, $138.1\left(\mathrm{C}-5^{\prime}\right), 146.3\left(\mathrm{C}-4^{\prime}\right), 155.0(\mathrm{C}-6 \mathrm{a}), 159.76\left(\mathrm{C}-4^{\prime \prime}\right), 159.75$ (C5a), 164.3 and 165.4 (C-1 and C-3), 174.3 (C-11) ppm. HRMS $\left(\mathrm{ESI}^{+}\right.$): $m / z$ calcd. for $\mathrm{C}_{26} \mathrm{H}_{19} \mathrm{~N}_{4} \mathrm{O}_{5}[\mathrm{M}+\mathrm{H}]^{+} 467.1355$, found 467.1332. MS $\left(\mathrm{ESI}^{+}\right): m / z(\%)=467(100)[\mathrm{M}+\mathrm{H}]^{+}, 489$ (27) $[\mathrm{M}$ $+\mathrm{Na}]^{+}, 505(20)[\mathrm{M}+\mathrm{K}]^{+}$.

4-[4-(4-Bromophenyl)-2-methyl-2H-1,2,3-triazol-5-yl]-2-methylchromeno[3,2-e]isoindole-1,3,11(2H)-trione (13d): Yield $52.3 \mathrm{mg}$ (57\%), m.p. $312-313{ }^{\circ} \mathrm{C} .{ }^{1} \mathrm{H}$ NMR $\left(500 \mathrm{MHz}, \mathrm{CDCl}_{3}\right): \delta=3.09$ (s, $\left.3 \mathrm{H}, 2-\mathrm{NCH}_{3}\right), 4.34\left(\mathrm{~s}, 3 \mathrm{H}, 2^{\prime}-\mathrm{NCH}_{3}\right), 7.29(\mathrm{~d}, J=8.6 \mathrm{~Hz}, 2 \mathrm{H}$, $\left.2^{\prime \prime}, 6^{\prime \prime}-\mathrm{H}\right), 7.42$ (d, $\left.J=8.6 \mathrm{~Hz}, 2 \mathrm{H}, 3^{\prime \prime}, 5^{\prime \prime}-\mathrm{H}\right), 7.47$ (ddd, $J=8.0$, 7.1, 0.7 Hz, $1 \mathrm{H}, 9-\mathrm{H}), 7.51(\mathrm{dd}, J=8.3,0.7 \mathrm{~Hz}, 1 \mathrm{H}, 7-\mathrm{H}), 7.79$ (ddd, $J=8.3,7.1,1.6 \mathrm{~Hz}, 1 \mathrm{H}, 8-\mathrm{H}), 7.84$ (s, $1 \mathrm{H}, 5-\mathrm{H}), 8.40$ (dd, $J=8.0,1.6 \mathrm{~Hz}, 1 \mathrm{H}, 10-\mathrm{H}) \mathrm{ppm} .{ }^{13} \mathrm{C}$ NMR $\left(126 \mathrm{MHz}, \mathrm{CDCl}_{3}\right): \delta$ $=24.5\left(2-\mathrm{NCH}_{3}\right), 42.2\left(2^{\prime}-\mathrm{NCH}_{3}\right), 117.6(\mathrm{C}-7), 119.8(\mathrm{C}-11 \mathrm{a}), 122.7$ (C-4' $\left.{ }^{\prime \prime}\right), 123.0$ (C-10a), 125.3 (C-9), 126.3 (C-5), 127.2 (C-3a), 127.5 (C-10), $128.4\left(\mathrm{C}-2^{\prime \prime}, 6^{\prime \prime}\right), 129.4\left(\mathrm{C}-1^{\prime \prime}\right), 132.0\left(\mathrm{C}-3^{\prime \prime}, 5^{\prime \prime}\right), 133.8$ and 134.1 (C-4 and C-11b), 135.6 (C-8), 138.7 (C-5'), 145.5 (C-4'), 155.0 (C-6a), 159.8 (C-5a), 164.1 and 165.4 (C-1 and C-3), 174.1 (C-11) ppm. HRMS (ESI $\left.{ }^{+}\right): m / z$ calcd. for $\mathrm{C}_{25} \mathrm{H}_{16}{ }^{79} \mathrm{BrN}_{4} \mathrm{O}_{4}[\mathrm{M}+$ $\mathrm{H}]^{+}$515.0355, found 515.0330; calcd. for $\mathrm{C}_{25} \mathrm{H}_{16}{ }^{81} \mathrm{BrN}_{4} \mathrm{O}_{4}[\mathrm{M}+$ $\mathrm{H}]^{+}$517.0334, found 517.0484. MS $\left(\mathrm{ESI}^{+}\right): m / z(\%)=515(15)[\mathrm{M}$ $+\mathrm{H}]^{+}, 537(24)[\mathrm{M}+\mathrm{Na}]^{+}, 555(21)[\mathrm{M}+\mathrm{K}]^{+}$.

\section{Acknowledgments}

Thanks are due to the Universidade de Aveiro, Instituto Politécnico de Bragança, the Fundação para a Ciência e Tecnologia (FCT) (PhD grant to H. M. T. A., SFRH/BD/86277/2012), the European Union (EU), the Quadro de Referência Estratégico Nacional (QREN), the Fundo Europeu de Desenvolvimento Regional (FEDER and COMPETE) for funding the QOPNA Research Unit (project number PEst-C/QUI/UI0062/2013) and the Portuguese National NMR Network.

[1] a) G. J. Bennett, H.-H. Lee, Phytochemistry 1989, 28, 967-998; b) S.-I. Sakai, M. Katsura, H. Takayama, N. Aimi, N. Chokethaworn, M. Suttajit, Chem. Pharm. Bull. 1993, 41, 958-960.

[2] T. B. P. Oldenburg, H. Wilkes, B. Horsfield, A. C. T. van Duin, D. Stoddart, A. Wilhelms, Org. Geochem. 2002, 33, 595-609.

[3] L. M. Vieira, A. Kijjoa, Curr. Med. Chem. 2005, 12, 2413 2446.

[4] a) H. R. El-Seedi, M. A. El-Barbary, D. M. El-Ghorab, L. Bohlin, A. K. Borg-Karlson, U. Goransson, R. Verpoorte, Curr. Med. Chem. 2010, 17, 854-901; b) M. M. Pinto, M. E. Sousa, M. S. Nascimento, Curr. Med. Chem. 2005, 12, $2517-$ 2538.

[5] a) K. H. Park, Y.-D. Park, J.-M. Han, K.-R. Im, B. W. Lee, I. Y. Jeong, T.-S. Jeong, W. S. Lee, Bioorg. Med. Chem. Lett. 2006, 16, 5580-5583; b) D. J. Jiang, Z. Dai, Y. J. Li, Cardiovasc. Drug Rev. 2004, 22, 91-102.

[6] a) Y. Na, J. Pharm. Pharmacol. 2009, 61, 707-712; b) G. C. Ee, S. Daud, S. A. Izzaddin, M. Rahmani, J. Asian Nat. Prod. Res. 2008, 10, 475-479.
[7] a) M. Riscoe, J. X. Kelly, R. Winter, Curr. Med. Chem. 2005 , 12, 2539-2549; b) C. Portela, C. M. M. Afonso, M. M. M. Pinto, M. J. Ramos, Bioorg. Med. Chem. 2004, 12, 3313-3321.

[8] a) C. M. M. Santos, M. Freitas, D. Ribeiro, A. Gomes, A. M. S. Silva, J. A. S. Cavaleiro, E. Fernandes, Bioorg. Med. Chem. 2010, 18, 6776-6784; b) W. Pothitirat, M. T. Chomnawang, R. Supabphol, W. Gritsanapan, Fitoterapia 2009, 80, 442-447.

[9] M. T. Khan, I. Orhan, F. S. Senol, M. Kartal, B. Sener, M. Dvorska, K. Smejkal, T. Slapetova, Chem.-Biol. Interact. 2009, 181, 383-389.

[10] a) R. Herbrecht, Int. J. Clin. Pract. 2004, 58, 612-624; b) S. Arikan, J. H. Rex, Curr. Opin. Investig. Drugs 2002, 3, 555-561; c) P. H. Chandrasekar, E. Manavathu, Drugs Today 2001, 37, $135-148$

[11] F. d. C. da Silva, M. C. B. V. de Souza, I. I. P. Frugulhetti, H. C. Castro, S. L. d. O. Souza, T. M. L. de Souza, D. Q. Rodrigues, A. M. T. Souza, P. A. Abreu, F. Passamani, C. R. Rodrigues, V. F. Ferreira, Eur. J. Med. Chem. 2009, 44, 373-383.

[12] K.-M. Alexacou, J. M. Hayes, C. Tiraidis, S. E. Zographos, D. D. Leonidas, E. D. Chrysina, G. Archontis, N. G. Oikonomakos, J. V. Paul, B. Varghese, D. Loganathan, Proteins Struct., Funct., Bioinf. 2008, 71, 1307-1323.

[13] M. J. Genin, D. A. Allwine, D. J. Anderson, M. R. Barbachyn, D. E. Emmert, S. A. Garmon, D. R. Graber, K. C. Grega, J. B. Hester, D. K. Hutchinson, J. Morris, R. J. Reischer, C. W. Ford, G. E. Zurenko, J. C. Hamel, R. D. Schaadt, D. Stapert, B. H. Yagi, J. Med. Chem. 2000, 43, 953-970.

[14] A. Massarotti, S. Aprile, V. Mercalli, E. Del Grosso, G. Grosa, G. Sorba, G. C. Tron, ChemMedChem 2014, 9, 2497-2508.

[15] N. H. Morgan, vol. EP 437979 A2, 1991, 0724.

[16] W. Fan, Comprehensive Heterocyclic Chem. II, vol. 4, Pergamon, Oxford, UK, UK, 1996.

[17] R. J. Willis, I. D. Marlow, Eur. Pat. Appl. 400842, 1990; Chem. Abstr. 1991, 114, 164247b.

[18] Y. Zou, Q. Zhao, H. Hu, L. Hu, S. Yu, M. Xu, Q. Wu, Arch. Pharmacol. Res. 2012, 35, 2093-2104.

[19] J. Li, M. Hu, S. Q. Yao, Org. Lett. 2009, 11, 3008-3011.

[20] W. A. Price, A. M. S. Silva, J. A. S. Cavaleiro, Heterocycles 1993, 36, 2601-2612.

[21] a) R. M. Letcher, T. Y. Yue, J. Chem. Res. Synop. 1992, 248; b) R. M. Letcher, T. Y. Yue, J. Chem. Res. Miniprint 1992, 2078.

[22] A. S. Kelkar, R. M. Letcher, K.-K. Cheung, K.-F. Chiu, G. D. Brown, J. Chem. Soc. Perkin Trans. 1 2000, 3732-3741.

[23] D. C. G. A. Pinto, A. M. S. Silva, C. M. Brito, A. Sandulache, J. R. Carrillo, P. Prieto, A. Díaz-Ortiz, A. de la Hoz, J. A. S. Cavaleiro, Eur. J. Org. Chem. 2005, 2973-2986.

[24] C. M. M. Santos, A. M. S. Silva, J. A. S. Cavaleiro, Eur. J. Org. Chem. 2009, 2642-2660.

[25] C. I. C. Esteves, C. M. M. Santos, C. M. Brito, A. M. S. Silva, J. A. S. Cavaleiro, Synlett 2011, 1403-1406.

[26] a) W. Baker, J. Chem. Soc. 1933, 1381-1389; b) H. S. Mahal, K. Venkataraman, J. Chem. Soc. 1934, 1767-1769.

[27] K. Sonogashira, Y. Tohda, N. Hagihara, Tetrahedron Lett. 1975, 16, 4467-4470.

[28] V. L. M. Silva, A. M. S. Silva, D. C. G. A. Pinto, J. Elguero, J. A. S. Cavaleiro, Eur. J. Org. Chem. 2009, 4468-4479.

[29] S. Kotha, S. Banerjee, RSC Adv. 2013, 3, 7642-7666.

[30] V. L. M. Silva, A. M. S. Silva, D. C. G. A. Pinto, J. A. S. Cavaleiro, Synlett 2006, 1369-1373.

[31] D. H. Wadsworth, S. M. Geer, M. R. Detty, J. Org. Chem. 1987, 52, 3662-3668.

[32] E. V. Tretyakov, A. V. Tkachev, T. V. Rybalova, Y. V. Gatilov, D. W. Knight, S. F. Vasilevsky, Tetrahedron 2000, 56, 10075 10080 .

Received: April 7, 2015 Published Online: June 10, 2015 